\title{
Perfectly Matched Layer Method for Acoustic Scattering Problem by a Locally Perturbed Line with Impedance Boundary Condition
}

\author{
Xue Jiang ${ }^{1, *}$ and Xujing $\mathrm{Li}^{2}$ \\ ${ }^{1}$ College of Applied Sciences, Beijing University of Technology, Beijing 100124, China \\ 2 Department of Mathematics, Hangzhou Normal University, Hangzhou, Zhejiang 310036, \\ China
}

Received 20 February 2019; Accepted (in revised version) 6 August 2019

\begin{abstract}
In this paper, we study the two-dimensional Helmholtz scattering problem by a locally perturbed line with impedance boundary condition. Different from the problem with Dirichlet boundary condition, the Green function of the Helmholtz equation with impedance boundary condition becomes very complicated and comprises surface waves along the locally perturbed line. A uniaxial perfectly matched layer (UPML) method is proposed to truncate the half plane into a bounded computational domain. The main contribution of this paper is to prove the well-posedness of the PML problem and the exponential convergence of the approximate solution to the exact solution as either the thickness or the medium parameter of PML increases.
\end{abstract}

AMS subject classifications: 65N30, 78A45, 35Q60

Key words: Uniaxial perfectly matched layer, Helmholtz equation, locally perturbed half-plane, impedance condition.

\section{Introduction}

Numerical solution of scattering problems has drawn considerable attentions for its broad real-life applications and mathematical interests. The treatment of radiation conditions of scattering solutions is the first key ingredient of numerical simulations. It involves the truncation of an unbounded domain to a bounded domain and imposes highly accurate boundary conditions at the artificial boundary (cf. e.g., [24-26, 34]). Recently, there arises a surge of studies on the scattering problems involving infinite boundaries, such as the scattering in layered media and half-spaces (cf. e.g., [11,19,20,22,35]). With the appearance of infinite boundaries, the scattering waves usually comprise reflective waves,

*Corresponding author.

Emails: jxue@lsec.cc.ac.cn (X. Jiang), lixujing@lsec.cc.ac.cn (X. J. Li) 
transmitted waves and surface waves. Thus the numerical treatment of radiation conditions becomes very challenging and appeals for new theories and methods.

In this paper, we study the time-harmonic scattering problem governed by the Helmholtz equation with impedance boundary condition in a locally perturbed halfplane:

$$
\begin{array}{ll}
\Delta u+k^{2} u=0 & \text { in } \mathbb{R}_{\Sigma+}^{2}, \\
\frac{\partial u}{\partial n}-\mathbf{i} k \beta u=g & \text { on } \Sigma, \\
\lim _{r=|x| \rightarrow+\infty} \int_{S_{r}^{1}}\left|\frac{\partial u}{\partial r}-\mathbf{i} k u\right|^{2}=0, & \lim _{r \rightarrow+\infty} \int_{S_{r}^{2}}\left|\frac{\partial u}{\partial r}-\mathbf{i} \sqrt{Z^{2}+k^{2}} u\right|^{2}=0,
\end{array}
$$

which could model outdoor sound propagation or the harbour resonances. For example, in the harbour resonances, the sea is supposed to fill the half-plane which is locally perturbed by harbour geometry. Here $k>0$ is the constant wave number, $\Sigma=\left\{\left(x_{1}, p\left(x_{1}\right)\right)\right.$ : $\left.x_{1} \in \mathbb{R}\right\}$ is the infinite boundary, $p \in C(\mathbb{R})$ is a piecewise $C^{1}$-smooth function supported in $[-1,1], \mathbb{R}_{\Sigma+}^{2}:=\left\{x \in \mathbb{R}^{2}: x_{2}>p\left(x_{1}\right)\right\}$ and $n$ is the unit outer normal on $\Sigma$ pointing to the exterior of $\mathbb{R}_{\Sigma+}^{2}$. For convenience, we write $\Sigma$ into the combination of the flat part and the perturbed part

$$
\Sigma=\Sigma_{\infty} \cup \Sigma_{p}, \quad \Sigma_{\infty}:=\left\{\left(x_{1}, 0\right):\left|x_{1}\right| \geq 1\right\}, \quad \Sigma_{p}=\Sigma \backslash \Sigma_{\infty} .
$$

Clearly $\Sigma$ is a local perturbation of the horizontal axis $\Sigma_{0}:=\partial \mathbb{R}_{+}^{2}$, where $\mathbb{R}_{+}^{2}:=\left\{\left(x_{1}, x_{2}\right)\right.$ : $\left.x_{1} \in \mathbb{R}, x_{2}>0\right\}$ (see Fig. 1 for an illustration of the setting). $\beta$ is the acoustic admittance, which would have to be taken as a complex-valued piecewise constant function in order to model the boundaries of rocks, sand, concrete in the applications. Here $\beta \in L^{\infty}(\Sigma)$ and satisfies

$$
\operatorname{Re}(\beta) \geq 0 \text { and } \beta \equiv-\mathbf{i} Z / k \text { on } \Sigma_{\infty},
$$

where $Z>0$ is the constant impedance parameter. We assume that the boundary condition on $\Sigma$ satisfies

$$
g \in H^{-1 / 2}(\Sigma), \quad \operatorname{supp}(g) \subset \Sigma_{p} .
$$

The two radiation conditions in (1.1c) represent propagating waves and surface waves respectively (see [22]) where

$$
S_{r}:=\left\{x \in \mathbb{R}_{+}^{2}:|x|=r\right\}, \quad S_{r}^{1}:=\left\{x \in S_{r}: x_{2} \geq r^{1 / 4}\right\}, \quad S_{r}^{2}:=S_{r} \backslash S_{r}^{1} .
$$

The existence and uniqueness of the solution to (1.1) are studied in [22]. Our concerns here are to propose an approximation of the radiation conditions on a truncation boundary and to solve the approximate problem on the bounded domain numerically. 


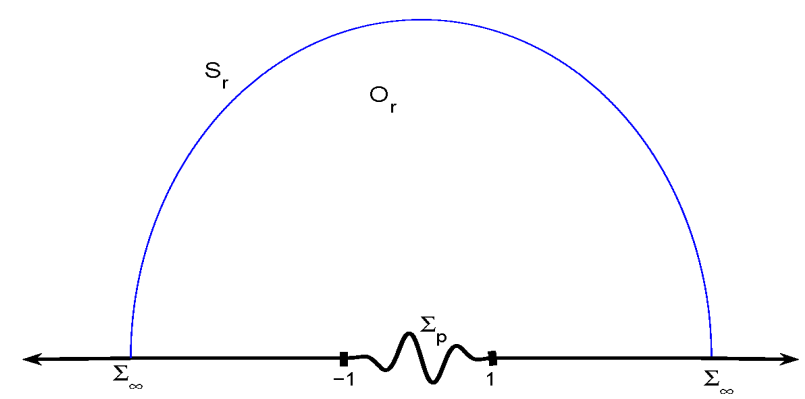

Figure 1: The setting of the scattering problem.

There are extensive studies on scattering problems in half-planes or half-spaces with local perturbations (cf e.g., [1-3,21-23,35] and the references therein). A practical application is the prediction of radar cross section of cavities in aircraft industries. In [1-3], Ammari and coauthors studied the well-posedness of electromagnetic scattering problems from cavities and proposed a variational method for numerical solutions of the problems. In [35], Zhang and coauthors proposed the UPML method for scattering problems from cavities in a half-plane. They studied the Helmholtz equation with Dirichlet boundary conditions on the infinite boundary $\Sigma$. The Green function in this case is the addition of two fundamental solutions of the Helmholtz equation which represent the point source and its image in the horizontal boundary $\Sigma_{0}$. In [22,23], Durán and coauthors studied the well-posedness of the scattering problems in locally perturbed half-plane and half-space with impedance boundary conditions on $\Sigma$. Different from the problem with Dirichlet boundary condition, the Green function of the Helmholtz equation with impedance boundary condition becomes very complicated and comprises surface waves which only propagate along $\Sigma$. The main objective of this paper is to propose a UPML method for solving (1.1) and to prove the exponential convergence of the UPML method.

Since the work of Bérénger [5], which proposed a PML method for solving the time dependent Maxwell equations, various constructions of PML absorbing layers have been proposed and studied in the literature (cf. e.g., Turkel and Yefet [33], Teixeira and Chew [32] for the reviews). The basic idea of the PML method is to surround the computational domain by a layer of finite thickness with specially designed model medium that absorbs all the waves that propagate from inside the computational domain. The convergence of the PML method for homogeneous background materials has drawn considerable attention in the literature. It is proven that the PML solution converges exponentially to the solution of the original scattering problem as the thickness of the PML layer tends to infinity. We refer to $[27,30,31]$ for circular PML methods for acoustic scattering problems. In 2005, Bao and Wu first proved the exponential convergence of the PML method for time-harmonic Maxwell's equations [4]. In [6-9], Bramble and Pasciak also studied the stability and exponential convergence of the PML method in both circular and Cartesian coordinates for acoustic and electromagnetic scattering problems. In [16,18], Chen 
and Zheng proved the exponential convergence of the PML method for time-harmonic acoustic and electromagnetic scattering problems in two-layer media. We also refer the reader to the papers $[10,17,28,29]$ on the PML methods for elastic scattering problems. In [12-15], Chen and coauthors developed the adaptive PML method for solving timeharmonic scattering problems. The method refines the mesh using a posteriori error estimate and produces a coarse mesh size away from the fixed domain such that the total computational cost is insensitive to the thickness of the PML absorbing layer.

The main objective of this paper is to prove the exponential convergence of UPML method for the scattering problem (1.1). Due to the impedance condition on the infinite boundary, the scattering solution comprises surface waves which only propagate in the horizontal direction and satisfy a nontraditional radiation condition. This makes the convergence analysis of the UPML method difficult. Our convergence proof is based on the Cagniard de-Hoop transformation of the Green function and the idea of the complex coordinate stretching. We first prove an integral representation of the solution of the exterior Helmholtz equation. Then using some elaborated estimation of the modified Green function, we show that the solution of the UPML problem converges exponentially to the solution of (1.1) as the thickness of the PML tends to infinity. In the literature, the work on investigating the exponential convergence of the PML method for scattering problems in locally perturbed half-plane with impedance conditions is very rare.

The layout of the paper is organized as follows. In Section 2 we study the Green function for the scattering problem in the upper half-plane. We use the Cagniard-de Hoop transform to derive an expression of the Green function which is crucial for the convergence analysis. In Section 3 we prove an integral representation of the solution to the scattering problem. In Section 4 we introduce the UPML formulation for (1.1) by the method of complex coordinate stretching. The exponential decay of the stretched solution is proved by estimating the stretched Green's function in the PML. In Section 5, we propose the PML approximation problem on the truncated domain and prove the well-posedness and exponential convergence of the problem. Additionally, some useful results concerning the PML transform are provided in the appendix.

\section{The Green function}

In this section we study the Green's function for the scattering problem in the upper half-plane

$$
\begin{cases}\Delta_{x} G(x, y)+k^{2} G(x, y)=-\delta_{y}(x) & \text { in } \mathbb{R}_{+}^{2}, \\ \frac{\partial G}{\partial x_{2}}(x, y)+Z G(x, y)=0 & \text { on } \Sigma_{0},\end{cases}
$$

where $\delta_{y}(\boldsymbol{x})$ is the Dirac source at $y \in \mathbb{R}_{+}^{2}$ and $\Sigma_{0}=\left\{\left(x_{1}, 0\right): x_{1} \in \mathbb{R}\right\}$. The Green function satisfying (2.1) is analyzed in [22] and is represented as an integral along an complex integral path. Here we derive the Green function for completeness and for our purpose of PML theory. First we will derive an explicit expression for the Green function by using 
the Fourier transform and the Sommerfeld Integral Path. Next we will use the Cagniardde Hoop transform to obtain a new formula of the Green function which will be crucial for us to prove the exponential decay of the PML extension in Section 4.

Throughout the paper we will always assume that for $z \in \mathbb{C}, z^{1 / 2}$ is the analytic branch of $\sqrt{z}$ such that $\operatorname{Re}\left(z^{1 / 2}\right) \geq 0$. This corresponds to the left half real axis as the branch cut in the complex plane. For $z=z_{1}+\mathbf{i} z_{2}, z_{1}, z_{2} \in \mathbb{R}$, we have

$$
z^{1 / 2}=\sqrt{\frac{|z|+z_{1}}{2}}+\mathbf{i} \operatorname{sgn}\left(z_{2}\right) \sqrt{\frac{|z|-z_{1}}{2}} .
$$

\subsection{The method of Fourier transform}

Write $G_{y}\left(x_{1}, x_{2}\right):=G(x, y)$ and let

$$
\hat{G}\left(\xi, x_{2}\right)=\frac{1}{\sqrt{2 \pi}} \int_{-\infty}^{\infty} G_{y}\left(x_{1}, x_{2}\right) e^{-\mathbf{i}\left(x_{1}-y_{1}\right) \xi} \mathrm{d} x_{1}
$$

be its Fourier transform for the first variable. By taking the Fourier transform of (2.1) in the first variable, we obtain the equation (with initial condition) in $x_{2}$ :

$$
\begin{cases}\frac{\partial^{2} \hat{G}}{\partial x_{2}^{2}}+\left(k^{2}-\xi^{2}\right) \hat{G}=-\frac{1}{\sqrt{2 \pi}} \delta_{y_{2}}\left(x_{2}\right) & \text { for } x_{2}>0 \\ \frac{\partial \hat{G}}{\partial x_{2}}+Z \hat{G}=0 & \text { for } x_{2}=0 .\end{cases}
$$

The solution of the initial problem (2.3) reads (see also [22])

$$
\hat{G}\left(\xi, x_{2}\right)=\frac{\mathbf{i}}{\sqrt{8 \pi}} \frac{1}{\mu}\left(\frac{Z-\mathbf{i} \mu}{Z+\mathbf{i} \mu} e^{\mathbf{i} \mu\left(x_{2}+y_{2}\right)}-e^{\mathbf{i} \mu\left|x_{2}-y_{2}\right|}\right),
$$

where $\mu$ is the square root defined by using the limiting absorption principle

$$
\mu(\xi)=\lim _{\varepsilon \rightarrow 0^{+}}\left[(k+\mathbf{i} \varepsilon)^{2}-\xi^{2}\right]^{1 / 2} .
$$

From (2.2), it is easy to see that, for $\xi \in \mathbb{R}$,

$$
\mu(\xi)= \begin{cases}\sqrt{k^{2}-\xi^{2}} & \text { if }|\xi| \leq k \\ \mathbf{i} \sqrt{\xi^{2}-k^{2}} & \text { if }|\xi|>k .\end{cases}
$$

Recall that the Green function for the Helmholtz equation is

$$
\Phi(x, y)=\frac{\mathbf{i}}{4} H_{0}^{(1)}(k|x-y|),
$$


which satisfies (cf. e.g., [19])

$$
\Phi(\boldsymbol{x}, \boldsymbol{y})=\frac{\mathbf{i}}{4 \pi} \int_{-\infty}^{+\infty} \frac{1}{\mu} e^{\mathbf{i}\left(x_{1}-y_{1}\right) \xi+\mathbf{i} \mu\left|x_{2}-y_{2}\right|} \mathrm{d} \xi .
$$

By taking the inverse Fourier transform of $\hat{G}\left(\xi, x_{2}\right)$, we obtain the Green's function

$$
\begin{aligned}
G(x, y) & =*-\Phi(x, y)+\Phi\left(x, y^{\prime}\right)+\frac{1}{2 \pi} \int_{-\infty}^{+\infty} \frac{1}{Z+\mathbf{i} \mu} e^{\mathrm{i}\left[\left(x_{1}-y_{1}\right) \xi+\left(x_{2}+y_{2}\right) \mu\right]} \mathrm{d} \xi \\
& =-\Phi(x, y)+\Phi\left(x, y^{\prime}\right)+\frac{1}{2 \pi} \int_{-\infty}^{+\infty} \frac{1}{Z+\mathbf{i} \mu} e^{\mathrm{i}\left[\left|x_{1}-y_{1}\right| \xi+\left(x_{2}+y_{2}\right) \mu\right]} \mathrm{d} \xi \\
& =-\Phi(x, y)+\Phi\left(x, y^{\prime}\right)+\frac{1}{2 \pi} I\left(1 /(Z+\mathbf{i} \mu) ;\left|x_{1}-y_{1}\right|, x_{2}+y_{2}\right),
\end{aligned}
$$

for any $\boldsymbol{x}, \boldsymbol{y} \in \mathbb{R}_{+}^{2}$, where $\boldsymbol{y}^{\prime}=\left(y_{1},-y_{2}\right)$ is the image of $\boldsymbol{y}=\left(y_{1}, y_{2}\right)$ in $\Sigma_{0}$ and

$$
I(h ; a, b):=\int_{-\infty}^{+\infty} h(\xi) e^{\mathrm{i}(a \xi+b \mu)} \mathrm{d} \xi, \quad \forall a, b>0 .
$$

Two possible roots of $Z+\mathbf{i} \mu(\xi)=0$ are

$$
\xi^{ \pm}=\lim _{\varepsilon \rightarrow 0^{+}} \xi_{\varepsilon}^{ \pm}, \quad \xi_{\varepsilon}^{ \pm}:= \pm\left[Z^{2}+(k+\mathbf{i} \varepsilon)^{2}\right]^{1 / 2} .
$$

While from (2.2) and (2.4), we have

$$
\mu\left(\xi^{ \pm}\right)=\lim _{\varepsilon \rightarrow 0^{+}} \mu\left(\xi_{\varepsilon}^{ \pm}\right)= \pm \mathbf{i} Z .
$$

Therefore, $Z+\mathbf{i} \mu(\xi)=0$ only has one $\operatorname{root} \xi^{+}$. The Green's function reads

$$
\begin{aligned}
G(x, y)=- & \Phi(x, y)+\Phi\left(x, y^{\prime}\right)-S(x, y) \\
& +\frac{1}{2 \pi} \text { P.V.I }\left(1 /(Z+\mathbf{i} \mu) ;\left|x_{1}-y_{1}\right|, x_{2}+y_{2}\right),
\end{aligned}
$$

where

$$
S(\boldsymbol{x}, \boldsymbol{y})=\frac{\mathbf{i} Z}{\sqrt{Z^{2}+k^{2}}} e^{-Z\left(x_{2}+y_{2}\right)} e^{\mathbf{i}\left|x_{1}-y_{1}\right| \sqrt{Z^{2}+k^{2}}} .
$$

Clearly $S(x, y)$ represents evanescent waves which propagate in the horizontal direction and decay exponentially in the vertical direction (see [22] for more discussions). From [22] we have the following theorem on the radiation condition for the Green function.

Theorem 2.1. Let $S_{r}^{1}, S_{r}^{2}$ be defined in (1.2) and $k_{Z}:=\sqrt{Z^{2}+k^{2}}$. Then for any $\boldsymbol{y} \in \mathbb{R}_{+}^{2}$, we have

$$
\begin{aligned}
& \lim _{r=|\boldsymbol{x}| \rightarrow+\infty} \int_{S_{r}^{1}}\left|\frac{\partial G(\boldsymbol{x}, \boldsymbol{y})}{\partial r}-\mathbf{i} k G(\boldsymbol{x}, \boldsymbol{y})\right|^{2} \mathrm{~d} S_{\boldsymbol{x}}=0, \\
& \lim _{r=|\boldsymbol{x}| \rightarrow+\infty} \int_{S_{r}^{2}}\left|\frac{\partial G(\boldsymbol{x}, \boldsymbol{y})}{\partial r}-\mathbf{i} k_{Z} G(\boldsymbol{x}, \boldsymbol{y})\right|^{2} \mathrm{~d} S_{\boldsymbol{x}}=0 .
\end{aligned}
$$




\subsection{The Cagniard-de Hoop transform}

Now we are going to derive a new form of the principal value integral P.V.I $(h ; a, b)$ by using the Cagniard-de Hoop (CdeH) transform [19, Page 215]. For any $\varepsilon>0$, the requirement that $\mu_{\varepsilon}(\xi):=\left[(k+\mathbf{i} \varepsilon)^{2}-\xi^{2}\right]^{1 / 2}$ has a positive imaginary part implies that the integral variable $\xi$ should be in the second and the fourth quadrants. Therefore, the principal value integral P.V.I $(h ; a, b)$ can be calculated along the Sommerfeld integral path (SIP)

$$
\text { P.V.I }(h ; a, b)=\text { P.V. } \int_{\text {SIP }} h(\xi) e^{\mathbf{i}(a \xi+b \mu)} \mathrm{d} \xi .
$$

We refer to [19, Chapter 2] for more discussion on the SIP (see Fig. 2).

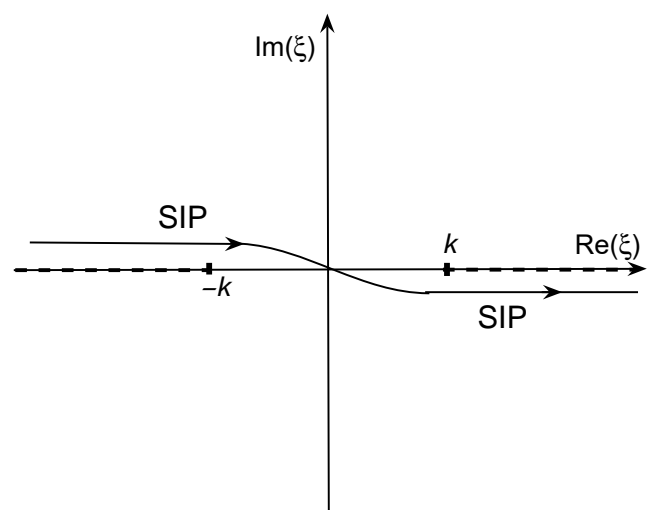

Figure 2: The Sommerfeld integral path.

Lemma 2.1. Let $a, b>0, \rho=\sqrt{a^{2}+b^{2}}$ and let $h$ be an analytic function in $\mathbb{C} \backslash((-\infty,-k] \cup[k, \infty))$ such that $\xi h(\xi)$ is bounded. Then

$$
\text { P.V.I }(h ; a, b)=-\mathbf{i} \int_{1}^{\infty} \frac{1}{\sqrt{t^{2}-1}}\left[(\mu h)\left(\xi_{+}(t)\right)+(\mu h)\left(\xi_{-}(t)\right)\right] e^{\mathbf{i} k \rho t} \mathrm{~d} t,
$$

where $\xi_{ \pm}$is defined by the CdeH transform

$$
\xi_{ \pm}(t)=k \rho^{-1}\left(a t \pm \mathbf{i} b \sqrt{t^{2}-1}\right), \quad \forall t \in[1,+\infty) .
$$

Proof. First we define a curve by the $\mathrm{CdeH}$ transform

$$
\Gamma=\Gamma_{+} \cap \Gamma_{-}, \quad \Gamma_{ \pm}:=\left\{\xi_{ \pm}: \xi_{ \pm}=k \rho^{-1}\left(a t \pm \mathbf{i} b \sqrt{t^{2}-1}\right), t \geq 1\right\}
$$

For any $\xi \in \Gamma$, write $\xi=\xi_{1}+\mathbf{i} \xi_{2}$ with $\xi_{1}, \xi_{2} \in \mathbb{R}$. Then

$$
\frac{\xi_{1}^{2}}{a^{2}}-\frac{\xi_{2}^{2}}{b^{2}}=\frac{k^{2}}{\rho^{2}}
$$




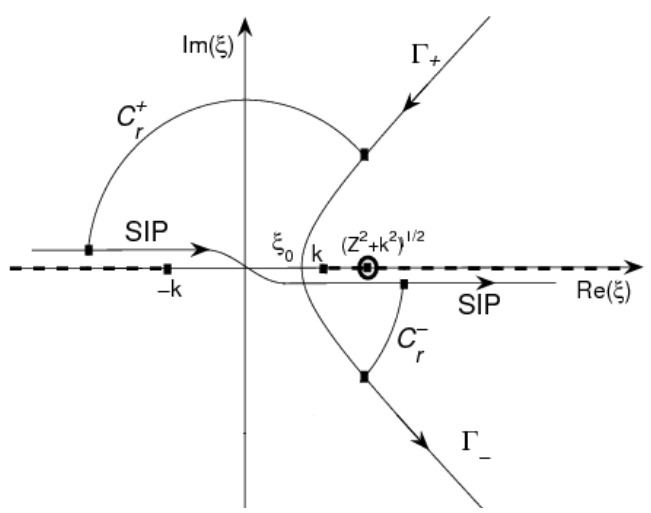

Figure 3: Cagniard-de Hoop transform from the SIP to $\Gamma_{+} \cup \Gamma_{-}$.

Clearly $\Gamma$ stands for the right branch of a hyperbola in the complex $\xi$-plane. The curve intersects the real axis at $\xi_{0}=k a / \rho$. By the convention in (2.2), we easily get

$$
\mu\left(\xi_{ \pm}\right)=\frac{k}{\rho}\left(b t \mp \mathbf{i} a \sqrt{t^{2}-1}\right), \quad \frac{\mathrm{d} \xi_{ \pm}}{\mathrm{d} t}= \pm \mathbf{i} \frac{\mu\left(\xi_{ \pm}\right)}{\sqrt{t^{2}-1}} .
$$

For any $r>0$, let $C_{r}^{+}, C_{r}^{-}$be respectively the part of the circle $\{\xi:|\xi|=r\}$ that are bounded by the SIP and $\Gamma_{+}$and by the SIP and $\Gamma_{-}$respectively. The geometry is depicted in Fig. 3. For the integrals on $C_{r}^{ \pm}$, we claim that

$$
\lim _{r \rightarrow \infty}\left|\int_{C_{r}^{ \pm}} h(\xi) e^{\mathbf{i}(\xi a+\mu b)} \mathrm{d} \xi\right|=0
$$

The proof of (2.11) will be postponed to the last two paragraphs of this proof. Using Cauchy integral theorem and letting $r \rightarrow \infty$, we obtain

$$
\text { P.V.I }(h ; a, b)=\text { P.V. } \int_{\text {SIP }} h(\xi) e^{\mathbf{i}(\xi a+\mu b)} \mathrm{d} \xi=\text { P.V. } \int_{\Gamma} h(\xi) e^{\mathbf{i}(\xi a+\mu b)} \mathrm{d} \xi,
$$

which by the definition of $\Gamma$ and (2.10) yields

$$
\text { P.V.I }(h ; a, b)=-\mathbf{i} \int_{1}^{\infty} \frac{1}{\sqrt{t^{2}-1}}\left[(\mu h)\left(\xi_{+}(t)\right)+(\mu h)\left(\xi_{-}(t)\right)\right] e^{\mathrm{i} k \rho t} \mathrm{~d} t .
$$

Now it is left to prove (2.11). We only prove the limit for the integral on $C_{r}^{+}$. The proof for the integral on $C_{r}^{-}$is similar and we omit the details. Let $C_{r}^{+}$intersect with $\Gamma_{+}$ at $\xi\left(\theta_{1}\right)=r e^{\mathbf{i} \theta_{1}}$ and with the $x$-axis at $\xi\left(\theta_{2}\right)=r e^{\mathbf{i} \theta_{2}}$. Clearly $0<\theta_{1}<\theta_{2} \leq \pi$. Since $\xi h(\xi)$ is bounded,

$$
\left|\int_{C_{r}^{+}} h(\xi) e^{\mathbf{i}(\xi a+\mu b)} \mathrm{d} \xi\right| \leq C \int_{\theta_{1}}^{\theta_{2}} e^{-\operatorname{Im}(\xi a+\mu b)} \mathrm{d} \theta .
$$


If $\theta \in\left(\theta_{1}, \pi / 2\right), \operatorname{Im} \mu^{2}=-r^{2} \sin 2 \theta<0$. From (2.2) we have

$$
\operatorname{Im}(\xi a+\mu b)=\operatorname{arsin} \theta-b\left[\frac{\left|k^{2}-r^{2} e^{2 \mathrm{i} \theta}\right|-\left(k^{2}-r^{2} \cos 2 \theta\right)}{2}\right]^{1 / 2},
$$

which is an increasing function in $\left[\theta_{1}, \pi / 2\right]$. Thus for $\theta_{1} \leq \theta \leq \pi / 2$,

$$
\operatorname{Im}(\xi a+\mu b) \geq \operatorname{Im}\left(\xi\left(\theta_{1}\right) a+\mu\left(\xi\left(\theta_{1}\right)\right) b\right)=k \rho t\left(\theta_{1}\right),
$$

where $\xi\left(\theta_{1}\right)=r e^{\mathbf{i} \theta_{1}} \in \Gamma_{+}$and $t\left(\theta_{1}\right) \in(1, \infty)$ satisfies

$$
r e^{\mathbf{i} \theta_{1}}=\frac{k}{\rho}\left[a t\left(\theta_{1}\right)+\mathbf{i} b \sqrt{t\left(\theta_{1}\right)^{2}-1}\right] .
$$

Therefore, we have $r \leq k t\left(\theta_{1}\right)$ and

$$
\operatorname{Im}(\xi a+\mu b) \geq k \rho t\left(\theta_{1}\right) \geq \rho r .
$$

If $\theta \in(\pi / 2,5 \pi / 6)$, since $\operatorname{Im} \mu^{2}=-r^{2} \sin 2 \theta>0$, we have

$$
\operatorname{Im}(\xi a+\mu b) \geq \operatorname{arsin} \theta>\operatorname{arsin}(5 \pi / 6)=\operatorname{ar} / 2 \text {. }
$$

If $\theta \in(5 \pi / 6, \pi), \operatorname{Re} \mu^{2}=k^{2}-r^{2} \cos 2 \theta<-r^{2} / 4$ for $r \gg k$. Then (2.2) shows that

$$
\operatorname{Im}(\xi a+\mu b) \geq b \operatorname{Im} \mu=b \sqrt{\frac{|\mu|^{2}-\operatorname{Re} \mu^{2}}{2}} \geq b r / 2 .
$$

Inserting (2.13)-(2.15) into (2.12) yields (2.11) for the integral on $C_{r}^{+}$.

\subsection{The modified Cagniard-de Hoop transform}

Notice that the $\mathrm{CdeH}$ transform $\xi_{ \pm}(t)$ defined in the previous section will degenerate as $b \rightarrow 0$. It makes the Green's function much more difficult to analyze. To solve this problem, we introduce a small constant $0<\delta<1 / 4$ and define

$$
b_{\delta}=\sqrt{b^{2}+\delta^{2} \rho^{2}}, \quad \rho_{\delta}=\sqrt{a^{2}+b_{\delta}^{2}}=\sqrt{1+\delta^{2}} \rho .
$$

The modified CdeH transform is defined by

$$
\Gamma_{\delta}=\left\{\xi_{ \pm}(t): 1 \leq t<+\infty\right\}, \quad \xi_{ \pm}(t)=k \rho_{\delta}^{-1}\left(a t \pm \mathbf{i} b_{\delta} \sqrt{t^{2}-1}\right)
$$

Now we define a new integral path by (see Fig. 4)

$$
\hat{\Gamma}=\left\{\xi \in \Gamma_{\delta}: \operatorname{Im} \xi>\operatorname{Im} \xi_{-}(\sqrt{2})\right\} \cup\left\{k t+\xi_{-}(\sqrt{2}): t \geq 0\right\} .
$$

Clearly, $\hat{\Gamma} \backslash \Gamma_{\delta}$ is located in the fourth quadrant. This indicates that $\mu_{ \pm}(t)$ are analytic functions in both the domain between $\hat{\Gamma} \cap \Gamma_{\delta}$ and the left half of the real axis and the domain between $\hat{\Gamma} \backslash \Gamma_{\delta}$ and the right half of the real axis (see Fig. 4). 


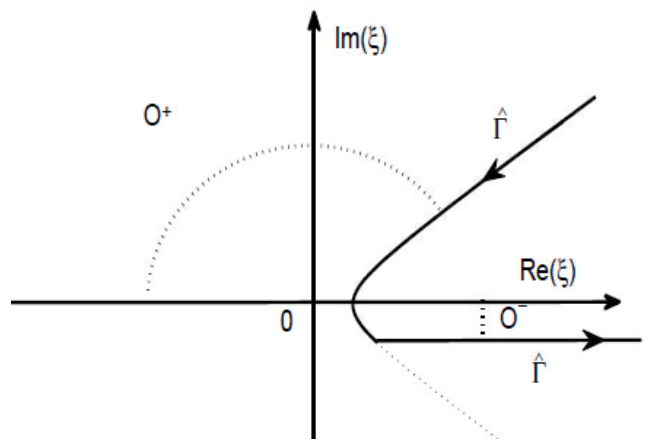

Figure 4: Cagniard-de Hoop transform from the SIP to $\hat{\Gamma}$.

Lemma 2.2. Let $a, b>0$ and let $h$ be an analytic function in $\mathbb{C} \backslash((-\infty,-k] \cup[k, \infty))$ such that $\xi h(\xi)$ is bounded. Then

$$
\begin{aligned}
\text { P.V.I }(h ; a, b)=- & \mathbf{i} \int_{1}^{\infty} \frac{F_{+}(t)}{\sqrt{t^{2}-1}} e^{\mathrm{i} k \rho_{\delta} t} \mathrm{~d} t-\mathbf{i} \int_{1}^{\sqrt{2}} \frac{F_{-}(t)}{\sqrt{t^{2}-1}} e^{\mathrm{i} k \rho_{\delta} t} \mathrm{~d} t \\
& +k e^{\mathbf{i} \sqrt{2} k \rho_{\delta}} \int_{0}^{\infty} G\left(k t+\xi_{-}(\sqrt{2})\right) e^{\mathbf{i} k a t} \mathrm{~d} t,
\end{aligned}
$$

where $\xi_{ \pm}$is defined by (2.8) and define

$$
\begin{aligned}
& \Lambda_{ \pm}(t)=k \rho_{\delta}^{-1}\left(b_{\delta} t \mp \mathbf{i} a \sqrt{t^{2}-1}\right), \\
& F_{ \pm}(t)=h\left(\xi_{ \pm}(t)\right) \Lambda_{ \pm}(t) e^{\mathbf{i}\left(b-b_{\delta}\right) \Lambda_{ \pm}(t)}, \quad G(\xi)=h(\xi) e^{\mathbf{i}\left[b \mu(\xi)-b_{\delta} \Lambda_{-}(\sqrt{2})\right]} .
\end{aligned}
$$

Proof. For any $r>0$, let $\mathrm{O}^{+}, \mathrm{O}^{-}$be respectively the part of the circle $\{\xi:|\xi|=r\}$ that are bounded by the SIP and $\hat{\Gamma}$ in the first quadrant and by the SIP and $\hat{\Gamma}$ in the fourth quadrant respectively. The geometry is depicted in Fig. 4. For the integrals on $\mathrm{O}^{+}$, by arguments similar to those in Lemma 2.1, we obtain

$$
\lim _{r \rightarrow \infty}\left|\int_{O^{+}} h(\xi) e^{\mathbf{i}(\tilde{\xi} a+\mu b)} \mathrm{d} \xi\right|=0
$$

For the integrals on $O^{-}$, since $\xi h(\xi)$ is bounded, $\operatorname{Im} \xi \geq \operatorname{Im} \xi_{-}(\sqrt{2})$ and $\operatorname{Im} \mu(\xi)>0$ in the fourth quadrant, there is a constant $C>0$ independent of $r$ such that

$$
\lim _{r \rightarrow \infty}\left|\int_{O^{-}} h(\xi) e^{\mathrm{i}(\xi a+\mu b)} \mathrm{d} \xi\right| \leq \lim _{r \rightarrow \infty} C r^{-1} \int_{O^{-}}|\xi h(\xi)| \mathrm{d} \xi=0 .
$$

Now using Cauchy integral theorem and letting $r \rightarrow \infty$, we obtain

$$
\text { P.V.I }(h ; a, b)=\text { P.V. } \int_{\text {SIP }} h(\xi) e^{\mathbf{i}(\xi a+\mu b)} \mathrm{d} \xi=\text { P.V. } \int_{\hat{\Gamma}} h(\xi) e^{\mathbf{i}(\xi a+\mu b)} \mathrm{d} \xi=I_{1}+I_{2},
$$


with

$$
I_{1}=\mathrm{P} . \mathrm{V} \cdot \int_{\hat{\Gamma} \cap \Gamma_{\delta}} h(\xi) e^{\mathbf{i}(\xi a+\mu b)} \mathrm{d} \xi, \quad I_{2}=\mathrm{P} . \mathrm{V} \cdot \int_{\hat{\Gamma} \backslash \Gamma_{\delta}} h(\xi) e^{\mathbf{i}(\xi a+\mu b)} \mathrm{d} \xi .
$$

By the definition of $\hat{\Gamma}$ and (2.16) yields

$$
I_{1}=-\mathbf{i} \int_{1}^{\infty} \frac{F_{+}(t)}{\sqrt{t^{2}-1}} e^{\mathrm{i} k \rho_{\delta} t} \mathrm{~d} t-\mathbf{i} \int_{1}^{\sqrt{2}} \frac{F_{-}(t)}{\sqrt{t^{2}-1}} e^{\mathrm{i} k \rho_{\delta} t} \mathrm{~d} t
$$

Moreover, since $\xi(t)=k t+\xi_{-}(\sqrt{2})$ for any $\xi \in \hat{\Gamma} \backslash \Gamma_{\delta}$ and $\sqrt{2} k \rho_{\delta}=a \xi_{-}(\sqrt{2})+b_{\delta} \Lambda_{-}(\sqrt{2})$, we have

$$
I_{2}=k e^{\mathbf{i} \sqrt{2} k \rho_{\delta}} \int_{0}^{\infty} h(\xi(t)) e^{\mathbf{i}\left[k a t+b \mu(\xi(t))-b_{\delta} \Lambda_{-}(\sqrt{2})\right]} \mathrm{d} t
$$

Then the proof is completed.

\subsection{The CdeH integral representation for the Green function}

We end up this section by applying Lemmas 2.1-2.2 to the Green function G. Let $0<\delta<$ $1 / 4$ be a constant which will be specified in Section 4 . Define

$$
\begin{aligned}
& a=\left|x_{1}-y_{1}\right|, \quad b=x_{2}+y_{2}, \quad \rho=\sqrt{a^{2}+b^{2}}, \\
& b_{\delta}=\sqrt{b^{2}+\delta^{2} \rho^{2}}, \quad \rho_{\delta}=\sqrt{a^{2}+\rho_{\delta}^{2}} .
\end{aligned}
$$

The CdeH transform is defined for $b>\delta a$ and $b \leq \delta a$ respectively by

$$
\begin{aligned}
& \xi_{ \pm}(t)=\left\{\begin{array}{ll}
\frac{k}{\rho}\left(a t \pm \mathbf{i} b \sqrt{t^{2}-1}\right), & \text { if } b>\delta a, \\
\frac{k}{\rho_{\delta}}\left(a t \pm \mathbf{i} b_{\delta} \sqrt{t^{2}-1}\right), & \text { if } b \leq \delta a,
\end{array} \quad \forall t \in[1,+\infty),\right. \\
& \Lambda_{ \pm}(t)=\left\{\begin{array}{ll}
\frac{k}{\rho}\left(b t \mp \mathbf{i} a \sqrt{t^{2}-1}\right), & \text { if } b>\delta a, \\
\frac{k}{\rho_{\delta}}\left(b_{\delta} \mp \mathbf{i} a \sqrt{t^{2}-1}\right), & \text { if } b \leq \delta a,
\end{array} \quad \forall t \in[1,+\infty) .\right.
\end{aligned}
$$

We define, if $b>\delta a$,

$$
J(h ; x, y)=-\mathbf{i} \int_{1}^{\infty}\left[h\left(\xi_{+}(t)\right) \Lambda_{+}(t)+h\left(\xi_{-}(t)\right) \Lambda_{-}(t)\right] \frac{e^{\mathbf{i} k \rho_{\delta} t}}{\sqrt{t^{2}-1}} \mathrm{~d} t
$$


and if $b \leq \delta a$,

$$
\begin{aligned}
J(h ; x, y)=- & \mathbf{i} \int_{1}^{\infty} h\left(\xi_{+}(t)\right) \Lambda_{+}(t) e^{\mathbf{i}\left(b-b_{\delta}\right) \Lambda_{+}(t)} \frac{e^{\mathbf{i} k \rho_{\delta} t}}{\sqrt{t^{2}-1}} \mathrm{~d} t \\
& -\mathbf{i} \int_{1}^{\sqrt{2}} h\left(\xi_{-}(t)\right) \Lambda_{-}(t) e^{\mathbf{i}\left(b-b_{\delta}\right) \Lambda_{-}(t)} \frac{e^{\mathbf{i} k \rho_{\delta} t}}{\sqrt{t^{2}-1}} \mathrm{~d} t \\
& +k e^{\mathbf{i} \sqrt{2} k \rho_{\delta}} \int_{0}^{\infty} G(\xi(t)) e^{\mathbf{i} k a t} \mathrm{~d} t,
\end{aligned}
$$

where $G(\xi)=h(\xi) e^{\mathbf{i}\left[b \mu(\xi)-b_{\delta} \Lambda_{-}(\sqrt{2})\right]}$ and $\xi(t)=k t+\xi_{-}(\sqrt{2})$.

Then the CdeH integral representation for the Green function is as follows

$$
\begin{aligned}
G(\boldsymbol{x}, \boldsymbol{y})=- & \frac{\mathbf{i}}{4 \pi} J\left(1 / \mu ;\left|x_{1}-y_{1}\right|,\left|x_{2}-y_{2}\right|\right)+\frac{\mathbf{i}}{4 \pi} J\left(1 / \mu ;\left|x_{1}-y_{1}\right|, x_{2}+y_{2}\right) \\
& +\frac{1}{2 \pi} J\left(1 /(Z+\mathbf{i} \mu) ;\left|x_{1}-y_{1}\right|, x_{2}+y_{2}\right)-S(\boldsymbol{x}, \boldsymbol{y}) .
\end{aligned}
$$

\section{The scattering problem}

The purpose of this section is to study the weak formulation of the scattering problem (1.1) and to show an integral representation of the scattering solution. We start by the well-posedness of (1.1). The following theorem can be proved by very similar arguments as in the proof of [22, Theorem 6.2]. We omit the details here.

Theorem 3.1. For any $g \in H^{-1 / 2}(\Sigma)$ satisfying $\operatorname{supp}(g) \subset \Sigma_{p}$, the scattering problem (1.1) has a unique solution $u \in H_{\mathrm{loc}}^{1}\left(\mathbb{R}_{\Sigma+}^{2}\right)$.

\subsection{An integral representation of the scattering solution}

Let $L_{1} \geq 1, L_{2}>\|p\|_{L^{\infty}(\mathbb{R})}$ be two constants and define

$$
\Omega_{1}=\left\{\boldsymbol{x} \in \mathbb{R}_{\Sigma+}^{2}:\left|x_{1}\right|<L_{1}, x_{2}<L_{2}\right\}, \quad \Gamma_{1}:=\partial \Omega_{1} \backslash \Sigma .
$$

Clearly $\Sigma_{p} \subset \partial \Omega_{1}$. Let $\Psi_{\mathrm{SL}}, \Psi_{\mathrm{DL}}$ be, respectively, the single-layer and double-layer potentials defined by

$$
\begin{array}{ll}
\Psi_{\mathrm{SL}}(\lambda)(\boldsymbol{x})=\int_{\Gamma_{1}} G(\boldsymbol{x}, \boldsymbol{y}) \lambda(\boldsymbol{y}) d S_{\boldsymbol{y}}, & \forall \lambda \in H^{-1 / 2}\left(\Gamma_{1}\right), \\
\Psi_{\mathrm{DL}}(g)(\boldsymbol{x})=\int_{\Gamma_{1}} \frac{\partial G(\boldsymbol{x}, \boldsymbol{y})}{\partial n_{y}} g(\boldsymbol{y}) d S_{\boldsymbol{y}}, & \forall g \in H^{1 / 2}\left(\Gamma_{1}\right) .
\end{array}
$$

Now we show an integral representation of the solution of the exterior scattering problem. It plays an important role in our subsequent analysis. 
Lemma 3.1. Any solution $u$ of the exterior problem (1.1) satisfies

$$
u=\Psi_{\mathrm{DL}}\left(\gamma_{D} u\right)-\Psi_{\mathrm{SL}}\left(\gamma_{D} u\right) \quad \text { in } \mathbb{R}_{\Sigma+}^{2} \backslash \bar{\Omega}_{1},
$$

where $\gamma_{D} u:=\left.u\right|_{\Gamma_{1}}$ and $\gamma_{N} u:=\left.\frac{\partial u}{\partial n}\right|_{\Gamma_{1}}$ are respectively the Dirichlet trace and the Neumann trace of $u$ on $\Gamma_{1}$.

Proof. First we introduce a circular truncation of $\mathbb{R}_{\Sigma+}^{2}$ by

$$
O_{r}=\left\{x \in \mathbb{R}_{\Sigma+}^{2}:|x|<r\right\}, \quad S_{r}=\partial O_{r} \backslash \Sigma, \quad \Sigma_{r}=\partial O_{r} \cap \Sigma .
$$

Let $r$ be large enough such that $\bar{\Omega}_{1} \subset O_{r}$. By Green's formula, we have

$$
u(\boldsymbol{x})=\int_{\Gamma_{1} \cup S_{r} \cup\left(\Sigma_{r} \backslash \Sigma_{1}\right)}\left[\frac{\partial u(\boldsymbol{y})}{\partial \boldsymbol{n}} G(\boldsymbol{x}, \boldsymbol{y})-\frac{\partial G(\boldsymbol{x}, \boldsymbol{y})}{\partial \boldsymbol{n}_{\boldsymbol{y}}} u(\boldsymbol{y})\right] \mathrm{d} S_{\boldsymbol{y}}, \quad \forall \boldsymbol{x} \in O_{R} \backslash \bar{\Omega}_{1},
$$

where $\boldsymbol{n}$ is the unit outer normal to $\partial\left(O_{r} \backslash \bar{B}_{1}\right)$. By the impedance boundary conditions in (1.1b) and (2.1), direct calculations show that

$$
\int_{\Sigma_{r} \backslash \Sigma_{1}}\left[\frac{\partial u(\boldsymbol{y})}{\partial \boldsymbol{n}} G(\boldsymbol{x}, \boldsymbol{y})-\frac{\partial G(\boldsymbol{x}, \boldsymbol{y})}{\partial n_{y}} u(\boldsymbol{y})\right] \mathrm{d} S_{y}=0, \quad \forall \boldsymbol{x} \in O_{r} \backslash \bar{\Omega}_{1} .
$$

Now it suffices to show that

$$
\int_{S_{r}}\left[\frac{\partial u(\boldsymbol{y})}{\partial \boldsymbol{n}} G(\boldsymbol{x}, \boldsymbol{y})-\frac{\partial G(\boldsymbol{x}, \boldsymbol{y})}{\partial n_{y}} u(\boldsymbol{y})\right] \mathrm{d} S_{y} \rightarrow 0 \quad \text { as } r \rightarrow \infty .
$$

To do that, we use the radiation conditions in (1.1c) and find that, as $R \rightarrow \infty$,

$$
\begin{aligned}
& \int_{S_{r}^{1}}\left[\left|\frac{\partial u}{\partial r}\right|^{2}+k^{2}|u|^{2}+2 k \operatorname{Im}\left(u \frac{\partial \bar{u}}{\partial r}\right)\right] \mathrm{d} S=\int_{S_{R}^{1}}\left|\frac{\partial u}{\partial r}-\mathbf{i} k u\right|^{2} \mathrm{~d} S \rightarrow 0, \\
& \int_{S_{r}^{2}}\left[\left|\frac{\partial u}{\partial r}\right|^{2}+k^{2}|u|^{2}+2 k_{Z} \operatorname{Im}\left(u \frac{\partial \bar{u}}{\partial r}\right)\right] \mathrm{d} S \rightarrow 0 .
\end{aligned}
$$

On the other hand, for any fixed $R_{0}<r$ such that $\bar{\Omega}_{1} \subset O_{R_{0}}$, using Green's formula again, we have

$$
\int_{\partial\left(O_{r} \backslash \bar{O}_{R_{0}}\right)} u \frac{\partial \bar{u}}{\partial n} \mathrm{~d} S=\int_{O_{r} \backslash \bar{O}_{R_{0}}}(u \Delta \bar{u}+\nabla u \cdot \nabla \bar{u}) \mathrm{d} x=\int_{O_{r} \backslash \bar{O}_{R_{0}}}\left(|\nabla u|^{2}-k^{2}|u|^{2}\right) \mathrm{d} x .
$$

Since

$$
u \frac{\partial \bar{u}}{\partial n}=Z|u|^{2}
$$

on $\Sigma_{\infty}$ by the impedance boundary condition, we have

$$
0=\operatorname{Im}\left(\int_{\partial\left(O_{r} \backslash \bar{O}_{R_{0}}\right)} u \frac{\partial \bar{u}}{\partial n} \mathrm{~d} S\right)=\operatorname{Im}\left(\int_{S_{R_{0}} \cup S_{r}} u \frac{\partial \bar{u}}{\partial n} \mathrm{~d} S\right),
$$


which indicates that

$$
\int_{S_{r}} \operatorname{Im}\left(u \frac{\partial \bar{u}}{\partial r}\right) \mathrm{d} s=\int_{S_{R_{0}}} \operatorname{Im}\left(u \frac{\partial \bar{u}}{\partial r}\right) \mathrm{d} s<\infty .
$$

Thus we deduce from (3.5a)-(3.5b) that $\|u\|_{L^{2}\left(S_{r}\right)}$ is bounded as $r \rightarrow \infty$.

Similarly, by Theorem 2.1 , we know that $\|G(\cdot, y)\|_{L^{2}\left(S_{r}\right)}$ is also bounded as $r \rightarrow \infty$. Note that

$$
\begin{aligned}
& \int_{S_{r}} {\left[\frac{\partial u(\boldsymbol{y})}{\partial \boldsymbol{n}} G(\boldsymbol{x}, \boldsymbol{y})-\frac{\partial G(\boldsymbol{x}, \boldsymbol{y})}{\partial n_{y}} u(\boldsymbol{y})\right] \mathrm{d} S_{y} } \\
&=\int_{S_{r}^{1}}\left[\left(\frac{\partial u(\boldsymbol{y})}{\partial n}-\mathbf{i} k u(\boldsymbol{y})\right) G(\boldsymbol{x}, \boldsymbol{y})-\left(\frac{\partial G(\boldsymbol{x}, \boldsymbol{y})}{\partial \boldsymbol{n}_{y}}-\mathbf{i} k G(\boldsymbol{x}, \boldsymbol{y})\right) u(\boldsymbol{y})\right] \mathrm{d} S_{y} \\
& \quad+\int_{S_{r}^{2}}\left[\left(\frac{\partial u(\boldsymbol{y})}{\partial n_{y}}-\mathbf{i} k_{Z} u(\boldsymbol{y})\right) G(\boldsymbol{x}, \boldsymbol{y})-\left(\frac{\partial G(\boldsymbol{x}, \boldsymbol{y})}{\partial n_{y}}-\mathbf{i} k_{Z} G(\boldsymbol{x}, \boldsymbol{y})\right) u(\boldsymbol{y})\right] \mathrm{d} S_{y} .
\end{aligned}
$$

Then (3.4) follows from (1.1c) and Theorem 2.1. This completes the proof.

Remark 3.1. From (3.2) and (2.6), the solution of (1.1) can be split into

$$
u(\boldsymbol{x})=u_{s}(\boldsymbol{x})+u_{p}(\boldsymbol{x}),
$$

where

$$
\begin{array}{rl}
u_{S}(\boldsymbol{x})=\int_{\Gamma_{1}} & {\left[S(\boldsymbol{x}, \boldsymbol{y}) \frac{\partial u(\boldsymbol{y})}{\partial n_{y}}-\frac{\partial S(\boldsymbol{x}, \boldsymbol{y})}{\partial \boldsymbol{n}_{y}} u(\boldsymbol{y})\right] \mathrm{d} S_{y},} \\
u_{p}(\boldsymbol{x})=\int_{\Gamma_{1}} & u(\boldsymbol{y}) \frac{\partial}{\partial n_{y}}\left[\frac{1}{2 \pi} \text { P.V.I }\left(\frac{1}{Z+\mathbf{i} \mu} ;\left|x_{1}-y_{1}\right|, x_{2}+y_{2}\right)-\Phi(\boldsymbol{x}, \boldsymbol{y})+\Phi\left(\boldsymbol{x}, \boldsymbol{y}^{\prime}\right)\right] \mathrm{d} S_{y} \\
& \quad-\int_{\Gamma_{1}} \frac{\partial u(\boldsymbol{y})}{\partial n_{y}}\left[\frac{1}{2 \pi} \text { P.V.I }\left(\frac{1}{Z+\mathbf{i} \mu} ;\left|x_{1}-y_{1}\right|, x_{2}+y_{2}\right)-\Phi(\boldsymbol{x}, \boldsymbol{y})+\Phi\left(\boldsymbol{x}, \boldsymbol{y}^{\prime}\right)\right] \mathrm{d} S_{y} .
\end{array}
$$

The first term $u_{s}$ stands for surface waves (or evanescent waves) which propagate in the horizontal direction but decay exponentially in the vertical direction. The second term $u_{p}$ stands for propagating waves in all directions of $\mathbb{R}_{+}^{2}$.

\subsection{A weak formulation of the scattering problem}

First we introduce the DtN operator $T: H^{1 / 2}\left(\Gamma_{1}\right) \rightarrow H^{-1 / 2}\left(\Gamma_{1}\right)$ be defined as follows: Given $f \in H^{1 / 2}\left(\Gamma_{1}\right), T f=\frac{\partial \chi}{\partial n}$ on $\Gamma_{1}$ where $\chi$ solves the scattering problem

$$
\begin{array}{ll}
\Delta \chi+k^{2} \chi=0 & \text { in } \mathbb{R}_{\Sigma+}^{2} \backslash \bar{\Omega}_{1}, \\
\frac{\partial \chi}{\partial x_{2}}+Z \chi=0 & \text { on } \Sigma \backslash \partial \Omega_{1}, \\
\chi=f & \text { on } \Gamma_{1}, \\
\lim _{r \rightarrow+\infty} \int_{S_{r}^{1}}\left|\frac{\partial \chi}{\partial r}-\mathbf{i} k \chi\right|^{2} \mathrm{~d} S=0, \quad \lim _{r \rightarrow+\infty} \int_{S_{r}^{2}}\left|\frac{\partial \chi}{\partial r}-\mathbf{i} \sqrt{Z^{2}+k^{2}} \chi\right|^{2} \mathrm{~d} S=0 .
\end{array}
$$




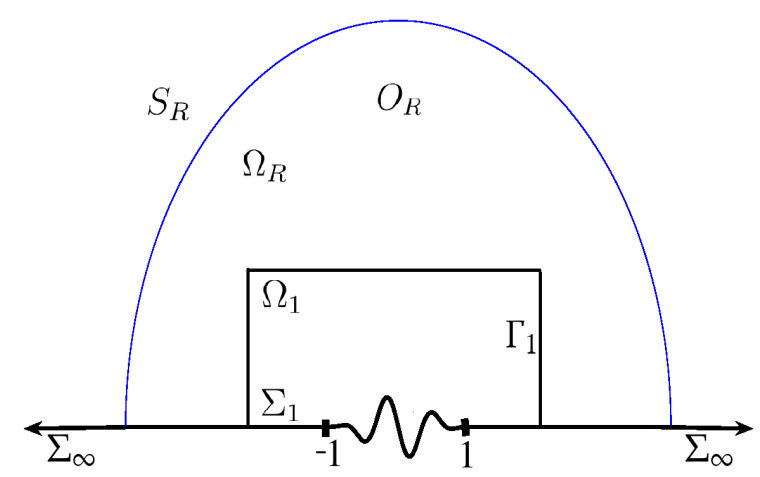

Figure 5: The setting of the scattering problem (3.7).

Let $R$ be sufficiently large such that $\bar{\Omega}_{1} \subset O_{R}$, where $O_{R}$ is defined by (3.3) and denote $\Omega_{R}=O_{R} \backslash \bar{\Omega}_{1}$ for convenience (see Fig. 5 for an illustration of the setting). We introduce the bilinear form $b: H^{1}\left(\Omega_{R}\right) \times H^{1}\left(\Omega_{R}\right) \rightarrow \mathbb{C}$ as follows:

$$
b(v, w)=\int_{\Omega_{R}}\left(\nabla v \cdot \nabla \bar{w}-k^{2} v \bar{w}\right) \mathrm{d} x-\left\langle D_{N} v, \bar{w}\right\rangle_{S_{R}}-Z\langle v, \bar{w}\rangle_{\Sigma_{R} \backslash \Sigma_{1}},
$$

where $\Sigma_{1}=\Sigma \cap \partial \Omega_{1}$ and $\langle\cdot, \cdot\rangle_{\Gamma}$ stands for the inner product on $L^{2}(\Gamma)$ or the duality pairing between $H^{-1 / 2}(\Gamma)$ and $H^{1 / 2}(\Gamma)$ for any one-dimensional Lipschitz manifold $\Gamma$. The DtN operator $D_{N}: H^{1 / 2}\left(S_{R}\right) \rightarrow H^{-1 / 2}\left(S_{R}\right)$ is defined as follows: Given $g \in H^{1 / 2}\left(S_{R}\right)$, define $D_{N}(g)=\frac{\partial \psi}{\partial n}$ on $S_{R}$, where $\psi$ solves

$$
\begin{array}{ll}
\Delta \psi+k^{2} \psi=0 & \text { in } \mathbb{R}_{\Sigma+}^{2} \backslash \bar{O}_{R}, \\
\frac{\partial \psi}{\partial x_{2}}+Z \psi=0 & \text { on } \Sigma \backslash \Sigma_{R}, \\
\psi=g & \text { on } S_{R}, \\
\lim _{r \rightarrow+\infty} \int_{S_{r}^{1}}\left|\frac{\partial \psi}{\partial r}-\mathbf{i} k \psi\right|^{2} \mathrm{~d} S=0, \quad \lim _{r \rightarrow+\infty} \int_{S_{r}^{2}}\left|\frac{\partial \psi}{\partial r}-\mathbf{i} \sqrt{Z^{2}+k^{2}} \psi\right|^{2} \mathrm{~d} S=0 .
\end{array}
$$

In [22, Section 6], Dúran and coauthors proved that (3.9) exists a unique solution, and showed the following property of $D_{N}$

$$
\|v\|_{L^{2}\left(S_{R}\right)}^{2} \leq-\operatorname{Re}\left\langle D_{N} v, \bar{v}\right\rangle_{S_{R}} \leq\|v\|_{H^{1 / 2}\left(S_{R}\right)}^{2} .
$$

A weak formulation of (3.7) reads: Find $\chi \in H^{1}\left(\Omega_{R}\right)$ such that $\chi=f$ on $\Gamma_{1}$ and

$$
b(\chi, v)=0, \quad \forall v \in H_{\Gamma_{1}}^{1}\left(\Omega_{R}\right):=\left\{w \in H^{1}\left(\Omega_{R}\right): w=0 \text { on } \Gamma_{1}\right\} .
$$

By using (3.10) and the trace inequality, we know that there exist two constants $\tau>0, \delta>0$ which only depend on $k, Z, \Omega_{R}$ such that

$$
b(v, v)+\tau\|v\|_{L^{2}\left(\Omega_{R}\right)}^{2} \geq \delta\|v\|_{H^{1}\left(\Omega_{R}\right)}^{2}, \quad \forall v \in H_{\Gamma_{1}}^{1}\left(\Omega_{R}\right) .
$$


By the spectral theory of compact operators, we deduce that (3.11) is a Fredholm equation and has a unique solution except possibly for a discrete set of values of $k$. Since our main objective is to study the PML approximation of (1.1), we do not elaborate on the wellposedness of the continuous problem (3.7) and merely assume that (3.7) has a unique solution for any $f \in H^{1 / 2}\left(\Gamma_{1}\right)$. Therefore $T$ is a well-defined linear continuous operator on $H^{1 / 2}\left(\Gamma_{1}\right)$.

Let the bilinear form $a: H^{1}\left(\Omega_{1}\right) \times H^{1}\left(\Omega_{1}\right) \rightarrow \mathbb{C}$ be defined by

$$
a(v, w)=\int_{\Omega_{1}}\left(\nabla v \cdot \nabla \bar{w}-k^{2} v \bar{w}\right) \mathrm{d} x-\langle T v, \bar{w}\rangle_{\Gamma_{1}}-\mathbf{i} k\langle\beta v, \bar{w}\rangle_{\Sigma_{1}} .
$$

From the continuity of $T$, we know that $a(\cdot, \cdot)$ is continuous on $H^{1}\left(\Omega_{1}\right) \times H^{1}\left(\Omega_{1}\right)$. A weak formulation of the scattering problem (1.1) reads: Find $u \in H^{1}\left(\Omega_{1}\right)$ such that

$$
a(u, v)=\langle g, \bar{v}\rangle_{\Sigma_{p}}, \quad \forall v \in H^{1}\left(\Omega_{1}\right) .
$$

Now Theorem 3.1 indicates that $a(\cdot, \cdot)$ is coercive on $H^{1}\left(\Omega_{1}\right)$, namely, satisfies the following inf-sup condition

$$
\sup _{0 \neq w \in H^{1}\left(\Omega_{1}\right)} \frac{|a(v, w)|}{\|w\|_{H^{1}\left(\Omega_{1}\right)}} \geq C_{\mathrm{IS}}\|v\|_{H^{1}\left(\Omega_{1}\right)}, \quad \forall v \in H^{1}\left(\Omega_{1}\right),
$$

where the constant $C_{\mathrm{IS}}>0$ only depends on $k, Z$ and $\Omega_{1}$.

\section{The uniaxial PML method}

Now we introduce the absorbing PML layer. Let $\alpha_{1}(t)=1+\mathbf{i} \sigma_{1}(t), \alpha_{2}(t)=1+\mathbf{i} \sigma_{2}(t)$ be the model medium property which satisfy

$$
\sigma_{j} \geq 0, \quad \sigma_{j}(t)=\sigma_{j}(-t) \quad \text { and } \quad \sigma_{j}=0 \quad \text { for }|t| \leq L_{j}, \quad j=1,2 .
$$

Denote by $\tilde{x}_{j}$ the complex coordinate stretching defined by

$$
\tilde{x}_{j}=\int_{0}^{x_{j}} \alpha_{j}(t) \mathrm{d} t, \quad j=1,2 .
$$

Notice that $\tilde{x}_{j}$ depends only on $x_{j}$ and for this reason the method is called the uniaxial PML method.

We extend the distance function by $r(\tilde{x}, \tilde{y})=\left[\left(\tilde{x}_{1}-\tilde{y}_{1}\right)^{2}+\left(\tilde{x}_{2}-\tilde{y}_{2}\right)^{2}\right]^{1 / 2}$ for complex variables and define the modified Green function by

$$
\tilde{G}(x, y):=G(\tilde{x}, \tilde{y}), \quad \forall x, y \in \mathbb{R}_{+}^{2} .
$$

For any $f \in H^{1 / 2}\left(\Gamma_{1}\right)$ and $\lambda \in H^{-1 / 2}\left(\Gamma_{1}\right)$, let the modified single-layer and double-layer potentials be defined by

$$
\widetilde{\Psi}_{\mathrm{SL}}(\lambda)(x):=\Psi_{\mathrm{SL}}(\lambda)(\tilde{\boldsymbol{x}}), \quad \widetilde{\Psi}_{\mathrm{DL}}(f)(x):=\Psi_{\mathrm{DL}}(f)(\tilde{\boldsymbol{x}}), \quad \forall x \in \mathbb{R}_{\Sigma+}^{2} \backslash \bar{\Omega}_{1} .
$$


For any $f \in H^{1 / 2}\left(\Gamma_{1}\right)$, we introduce the PML extension as follows

$$
\mathbb{E}(f)(\boldsymbol{x})=\widetilde{\Psi}_{\mathrm{DL}}(f)(\boldsymbol{x})-\widetilde{\Psi}_{\mathrm{SL}}(T f)(\boldsymbol{x}), \quad \forall x \in \mathbb{R}_{\Sigma+}^{2} \backslash \bar{\Omega}_{1} .
$$

Usually $\mathbb{E}$ is called the PML wave propagation operator. It is clear that $\mathbb{E}(f)$ is continuous and satisfies

$$
\gamma_{\Gamma_{1}} \mathbb{E}(f)=\gamma_{\Gamma_{1}} \tilde{\Psi}_{\mathrm{DL}}(f)-\gamma_{\Gamma_{1}} \tilde{\Psi}_{\mathrm{SL}}(T f)=\gamma_{\Gamma_{1}} \Psi_{\mathrm{DL}}(f)-\gamma_{\Gamma_{1}} \Psi_{\mathrm{SL}}(T f)=f,
$$

where $\gamma_{\Gamma_{1}}: H_{\text {loc }}^{1}\left(\mathbb{R}_{\Sigma+}^{2} \backslash \bar{\Omega}_{1}\right) \rightarrow H^{1 / 2}\left(\Gamma_{1}\right)$ is the trace operator.

Let $\tilde{u}(\boldsymbol{x})=u(\tilde{\boldsymbol{x}})$ be the PML extension of the solution $u$ of the scattering problem (3.13). It is obvious that $\tilde{u}$ satisfies

$$
\frac{\partial^{2} \tilde{u}}{\partial \tilde{x}_{1}^{2}}+\frac{\partial^{2} \tilde{u}}{\partial \tilde{x}_{2}^{2}}+k^{2} \tilde{u}=0 \quad \text { in } \mathbb{R}_{\Sigma+}^{2} \backslash \bar{\Omega}_{1},
$$

which yields the desired UPML equation by the chain rule

$$
\nabla \cdot(A \nabla \tilde{u})+\alpha_{1} \alpha_{2} k^{2} \tilde{u}=0 \quad \text { in } \mathbb{R}_{\Sigma+}^{2} \backslash \bar{\Omega}_{1},
$$

where $A=\operatorname{diag}\left(\alpha_{2}\left(x_{2}\right) / \alpha_{1}\left(x_{1}\right), \alpha_{1}\left(x_{1}\right) / \alpha_{2}\left(x_{2}\right)\right)$ is a diagonal matrix. From the DtN operator $T$ defined by (3.7) and the integral representation (3.1a), we have $\tilde{u}=\mathbb{E}\left(\left.u\right|_{\Gamma_{1}}\right)$ in $\mathbb{R}_{\Gamma+}^{2} \backslash \Omega_{1}$. The purpose of this section is to show that the PML extension $\mathbb{E}\left(\left.u\right|_{\Gamma_{1}}\right)$ (or $\tilde{u}$ ) will decay exponentially in the absorbing layer. We shall estimate the trace of $\mathbb{E}(f)$ on the truncated boundary for any $f \in H^{1 / 2}\left(\Gamma_{1}\right)$.

In the rest of this paper, we make the following assumption on the fictitious medium property which is rather mild in practical applications of the UPML method:

(H1) $\int_{0}^{L_{1}+d_{1}} \sigma_{1}(t) \mathrm{d} t=\int_{0}^{L_{2}+d_{2}} \sigma_{2}(t) \mathrm{d} t=: \bar{\sigma}, \bar{\sigma} \geq 1$ is a constant.

Remark 4.1. For instance, we may choose $\sigma_{1}(t)=\hat{\sigma}_{1}\left(\frac{|t|-L_{1}}{d_{1}}\right)^{m}, \sigma_{2}(t)=\hat{\sigma}_{2}\left(\frac{|t|-L_{2}}{d_{2}}\right)^{m}$ for $|t|>L_{j}$, where $m \geq 1$ is an integer and $\hat{\sigma}_{j}>0$ is a constant, $j=1,2$. Then $\bar{\sigma}$ can be enlarged by increasing the constants $\hat{\sigma}_{1}$ and $\hat{\sigma}_{2}$.

\subsection{Estimation of the modified Green function}

First we present some preliminary lemmas for the estimation of the modified Green function.

Lemma 4.1 ([15]). For any $\tilde{a}=a_{1}+\mathbf{i} a_{2}$ and $\tilde{b}=b_{1}+\mathbf{i} b_{2}$ with $a_{1}, a_{2}, b_{1}, b_{2} \in \mathbb{R}$ such that $a_{1} a_{2}+$ $b_{1} b_{2}>0$ and $a_{1}^{2}+b_{1}^{2}>0$, we have

$$
\operatorname{Im}\left(\tilde{a}^{2}+\tilde{b}^{2}\right)^{1 / 2} \geq \frac{a_{1} a_{2}+b_{1} b_{2}}{\sqrt{a_{1}^{2}+b_{1}^{2}}} .
$$


Let $\Omega_{2}=\left\{x \in \mathbb{R}_{\Sigma+}^{2}:\left|x_{1}\right|<L_{1}+d_{1}, x_{2}<L_{2}+d_{2}\right\}$ be the truncation domain which contains $\Omega_{1}$ and denote $\Sigma_{2}=\Sigma \cap \partial \Omega_{2}, \Gamma_{2}=\partial \Omega_{2} \backslash \Sigma_{2}$. In the following we will always denote

$$
\tilde{a}=\left[\left(\tilde{x}_{1}-y_{1}\right)^{2}\right]^{1 / 2}, \quad \tilde{b}=\tilde{x}_{2}+y_{2}, \quad \tilde{\rho}=\rho(\tilde{x}, y):=\left(\tilde{a}^{2}+\tilde{b}^{2}\right)^{1 / 2},
$$

for any $\boldsymbol{x} \in \Gamma_{2}, \boldsymbol{y} \in \bar{\Omega}_{1}$. From the convention (2.2), it is easy to see that $\tilde{a}=a_{1}+\mathbf{i} a_{2}, \tilde{b}=b_{1}+\mathbf{i} b_{2}$ with

$$
a_{1}=\left|x_{1}-y_{1}\right|, \quad a_{2}=\left|\int_{0}^{x_{1}} \sigma_{1}(t) \mathrm{d} t\right|, \quad b_{1}=x_{2}+y_{2}, \quad b_{2}=\int_{0}^{x_{2}} \sigma_{2}(t) \mathrm{d} t .
$$

By Lemma 4.1 and (4.5), $\tilde{\rho}=\rho(\tilde{x}, y)$ satisfy

$$
\operatorname{Im} \tilde{\rho} \geq \frac{a_{1} a_{2}+b_{1} b_{2}}{\sqrt{a_{1}^{2}+b_{1}^{2}}} \geq \gamma_{0} \bar{\sigma}, \quad \forall \boldsymbol{x} \in \Gamma_{2}, \quad \boldsymbol{y} \in \bar{\Omega}_{1},
$$

where we have used (H1) and $\gamma_{0}=\frac{\min \left(d_{1}, d_{2}\right)}{\sqrt{\left(2 L_{1}+d_{1}\right)^{2}+\left(2 L_{2}+d_{2}\right)^{2}}}$. We remark that $\gamma_{0} \sim \mathcal{O}(1)$ for $d_{1}$ and $d_{2}$ have the same scale.

Write $\tilde{\rho}=\rho_{1}+\mathbf{i} \rho_{2}$, then $\tilde{\rho}^{2}=\left(\rho_{1}^{2}-\rho_{2}^{2}+2 \mathbf{i} \rho_{1} \rho_{2}\right)$. Since $\tilde{\rho}^{2}=\tilde{a}^{2}+\tilde{b}^{2}=a_{1}^{2}-a_{2}^{2}+b_{1}^{2}-b_{2}^{2}+$ $2 \mathbf{i}\left(a_{1} a_{2}+b_{1} b_{2}\right)$, we have $\rho_{1} \rho_{2}=a_{1} a_{2}+b_{1} b_{2}$. Then by Lemma 4.1,

$$
\operatorname{Re} \tilde{\rho}=\rho_{1}=\frac{a_{1} a_{2}+b_{1} b_{2}}{\rho_{2}} \leq \sqrt{a_{1}^{2}+b_{1}^{2}} .
$$

Furthermore, we denote

$$
\tilde{b}_{\delta}=\left(\tilde{b}^{2}+\delta^{2} \tilde{\rho}^{2}\right)^{1 / 2}, \quad \tilde{\rho}_{\delta}=\left(\tilde{a}^{2}+\tilde{b}_{\delta}^{2}\right)^{1 / 2} .
$$

It's easy to see that

$$
\tilde{\rho}_{\delta}=\left(\tilde{a}^{2}+\tilde{b}^{2}+\delta^{2} \tilde{\rho}^{2}\right)^{1 / 2}=\sqrt{1+\delta^{2}} \tilde{\rho},
$$

and then

$$
\operatorname{Im} \tilde{\rho}_{\delta} \geq \sqrt{1+\delta^{2}} \gamma_{0} \bar{\sigma}, \quad \forall \boldsymbol{x} \in \Gamma_{2}, \quad \boldsymbol{y} \in \bar{\Omega}_{1} .
$$

The following lemma is the complex counterpart of (2.8) and (2.10).

Lemma 4.2. For any $\tilde{a}=a_{1}+\mathbf{i} a_{2}$ and $\tilde{b}=b_{1}+\mathbf{i} b_{2}$ with $a_{1}, a_{2}, b_{1}, b_{2} \geq 0$, define

$$
\xi_{ \pm}(t)=\left\{\begin{array}{ll}
\frac{k}{\tilde{\rho}}\left(\tilde{a} t \pm \mathbf{i} \tilde{b} \sqrt{t^{2}-1}\right), & \text { if } b_{1}>\delta a_{1}, \\
\frac{k}{\tilde{\rho}_{\delta}}\left(\tilde{a} t \pm \mathbf{i} \tilde{b}_{\delta} \sqrt{t^{2}-1}\right), & \text { if } b_{1} \leq \delta a_{1},
\end{array} \quad \forall t \in[1,+\infty) .\right.
$$


Then $\mu\left(\xi_{ \pm}\right)=\left(k^{2}-\xi_{ \pm}^{2}\right)^{1 / 2}$ satisfies

$$
\mu_{ \pm}(t)=\left\{\begin{array}{ll}
\frac{k}{\tilde{\rho}}\left(\tilde{b} t \mp \mathbf{i} \tilde{a} \sqrt{t^{2}-1}\right), & \text { if } b_{1}>\delta a_{1}, \\
\frac{k}{\tilde{\rho}_{\delta}}\left(\tilde{b}_{\delta} \mp \mathbf{i} \tilde{a} \sqrt{t^{2}-1}\right), & \text { if } b_{1} \leq \delta a_{1},
\end{array} \quad \forall t \in[1,+\infty) .\right.
$$

Proof. For any $t \in[1, \infty)$, let $\mu_{0}^{ \pm}=k\left(\tilde{b} t \mp \mathbf{i} \tilde{a} \sqrt{t^{2}-1}\right) / \tilde{\rho}$ for $b_{1}>\delta a_{1}$ and $\mu_{0}^{ \pm}=k\left(\tilde{b}_{\delta} t \mp\right.$ $\left.\mathbf{i} \tilde{a} \sqrt{t^{2}-1}\right) / \tilde{\rho}_{\delta}$ for $b_{1} \leq \delta a_{1}$. Clearly $\left(\mu_{0}^{ \pm}\right)^{2}=k^{2}-\tilde{\xi}_{ \pm}^{2}=\mu_{ \pm}^{2}$. The lemma follows from the convention (2.2) and $\operatorname{Re}\left(\mu_{0}^{ \pm}\right) \geq 0$ which can be proved by direct calculations. Here we omit the details.

In the following, we will always denote $\tilde{z}=z_{1}+\mathbf{i} z_{2}$ for $\tilde{z}=\tilde{a}, \tilde{b}, \tilde{\rho}$ and $\tilde{z}_{\delta}=z_{1}^{\delta}+\mathbf{i} z_{2}^{\delta}$ for $\tilde{z}_{\delta}=\tilde{b}_{\delta}, \tilde{\rho}_{\delta}$. Moreover, we make the following assumptions on the transform parameter $\delta$ and the PML medium parameter $\bar{\sigma}$ :

(H2) $0<\delta<\frac{\gamma_{0}^{2}}{20}$ and $\bar{\sigma} \geq 9\left(\frac{Z}{k}+1\right)^{3} \gamma_{0}^{-5}\left(2 L_{2}+d_{2}\right)$.

We remark that the above assumption of $\bar{\sigma}$ is only used for theoretical analysis. Practically, $\bar{\sigma}$ can be any constant large than one unit wavelength, while larger value of $\bar{\sigma}$ meaning faster decay of the scattering solution in the PML.

In the rest of the paper, without specifications, we let $C>0$ be a generic constant which may depend on $k$ and $Z$, but is independent of $\bar{\sigma}$ and $d=\max \left(d_{1}, d_{2}, L_{1}, L_{2}\right)$.

Lemma 4.3. Let $\tilde{a}, \tilde{b}, \tilde{\rho}, \tilde{b}_{\delta}$ and $\tilde{\rho}_{\delta}$ be defined in (4.5) and (4.8) for any $x \in \Gamma_{2}, y \in \bar{\Omega}_{1}$. For any $h \in L^{\infty}([1, \infty))$, define

$$
\begin{array}{ll}
F(h ; \tilde{a}, \tilde{b})=\frac{1}{2 \pi} \int_{1}^{\infty} \frac{1}{\sqrt{t^{2}-1}} h(t) e^{\mathrm{i} k \tilde{\rho} t} \mathrm{~d} t, & \text { if } b_{1}>\delta a_{1}, \\
F_{1}^{ \pm}(h ; \tilde{a}, \tilde{b})=\frac{1}{2 \pi} \int_{1}^{\infty} \frac{1}{\sqrt{t^{2}-1}} h\left(\mu_{ \pm}(t)\right) e^{\mathbf{i}\left(\tilde{b}-\tilde{b}_{\delta}\right) \mu_{ \pm}(t)} e^{\mathbf{i} k \tilde{\rho}_{\delta} t} \mathrm{~d} t, & \text { if } b_{1} \leq \delta a_{1} .
\end{array}
$$

Let (H1)-(H2) be satisfied. Then for any integer $n$,

$$
\begin{aligned}
& \left|F\left(t^{n} h ; \tilde{a}, \tilde{b}\right)\right| \leq C\|h\|_{L^{\infty}([1, \infty))} e^{-k \gamma_{0} \bar{\sigma}}, \\
& \left|F_{1}^{ \pm}\left(t^{n} h ; \tilde{a}, \tilde{b}\right)\right| \leq C\|h\|_{L^{\infty}([1, \infty))} e^{-\frac{1}{2} k \gamma_{0} \bar{\sigma}} .
\end{aligned}
$$

Proof. We only prove the lemma for $F_{1}^{ \pm}(h ; \tilde{a}, \tilde{b})$. The case of $F(h ; \tilde{a}, \tilde{b})$ is similar and easier, so we omit the details here.

First, we estimate $\mu_{ \pm}$. Noting that when $\rho(x, y) \geq 2 \bar{\sigma}$,

$$
|\rho(\tilde{x}, \boldsymbol{y})| \geq\left|\operatorname{Re}\left(\tilde{a}^{2}+\tilde{b}^{2}\right)\right|^{1 / 2} \geq\left(\rho(\boldsymbol{x}, \boldsymbol{y})^{2}-2 \bar{\sigma}^{2}\right)^{1 / 2} \geq \frac{1}{\sqrt{2}} \rho(\boldsymbol{x}, \boldsymbol{y}) .
$$


Then by (4.9),

$$
\left|\rho_{\delta}(\tilde{x}, y)\right|=\sqrt{1+\delta^{2}}|\rho(\tilde{x}, y)| \geq \frac{\sqrt{1+\delta^{2}}}{\sqrt{2}} \rho(x, y) .
$$

Thus for any $x \in \Gamma_{2}, y \in \bar{\Omega}_{1}$,

$$
\frac{|\tilde{a}|}{\left|\tilde{\rho}_{\delta}\right|}=\frac{\left|\tilde{x}_{1}-y_{1}\right|}{\left|\rho_{\delta}(\tilde{x}, \boldsymbol{y})\right|} \leq \frac{\sqrt{2}\left(\rho(x, y)^{2}+\bar{\sigma}^{2}\right)^{1 / 2}}{\sqrt{1+\delta^{2}} \rho(x, y)} \leq \frac{\sqrt{10}}{2 \sqrt{1+\delta^{2}}}
$$

and

$$
\frac{\left|\tilde{b}_{\delta}\right|}{\left|\tilde{\rho}_{\delta}\right|} \leq \frac{|\tilde{b}|}{\left|\tilde{\rho}_{\delta}\right|}+\frac{|\delta \tilde{\rho}|}{\left|\tilde{\rho}_{\delta}\right|}=\frac{\left|\tilde{x}_{2}+y_{2}\right|}{\left|\tilde{\rho}_{\delta}\right|}+\frac{\delta|\tilde{\rho}|}{\sqrt{1+\delta^{2}}|\tilde{\rho}|} \leq \frac{\sqrt{10}}{2 \sqrt{1+\delta^{2}}}+\frac{\delta}{\sqrt{1+\delta^{2}}}
$$

where we have written $\tilde{\rho}=\rho(\tilde{x}, \boldsymbol{y})$ and $\tilde{\rho}_{\delta}=\rho_{\delta}(\tilde{\boldsymbol{x}}, \boldsymbol{y})$ for simplicity.

When $\rho(\boldsymbol{x}, \boldsymbol{y}) \leq 2 \bar{\sigma},(4.10)$ yields that for any $\boldsymbol{x} \in \Gamma_{2}, \boldsymbol{y} \in \bar{\Omega}_{1}$,

$$
\frac{|\tilde{a}|}{\left|\tilde{\rho}_{\delta}\right|} \leq \frac{\left(\rho(\boldsymbol{x}, \boldsymbol{y})^{2}+\bar{\sigma}^{2}\right)^{1 / 2}}{\operatorname{Im} \tilde{\rho}_{\delta}} \leq \frac{\left(\rho(\boldsymbol{x}, \boldsymbol{y})^{2}+\bar{\sigma}^{2}\right)^{1 / 2}}{\sqrt{1+\delta^{2}} \gamma_{0} \bar{\sigma}} \leq \frac{\sqrt{5}}{\sqrt{1+\delta^{2}}} \gamma_{0}^{-1},
$$

and

$$
\frac{\left|\tilde{b}_{\delta}\right|}{\left|\tilde{\rho}_{\delta}\right|} \leq \frac{\sqrt{5}}{\sqrt{1+\delta^{2}}} \gamma_{0}^{-1}+\frac{\delta}{\sqrt{1+\delta^{2}}}
$$

Substitute the above estimates into (4.12), we have $\left|\mu_{ \pm}\right| \leq 5 \mathrm{kt} \gamma_{0}^{-1}$ for all $t \geq 1$.

Note that $b_{1} \leq \delta a_{1}$, it implies that $x_{2}<L_{2}$ and $b_{2}=0$. Then

$$
|\tilde{\rho}|=\left[\left(a_{1}^{2}-a_{2}^{2}+b_{1}^{2}-b_{2}^{2}\right)^{2}+4\left(a_{1} a_{2}+b_{1} b_{2}\right)^{2}\right]^{1 / 4} \leq\left[\bar{\sigma}^{4}+4 a_{1}^{2} \bar{\sigma}^{2}\right]^{1 / 4} .
$$

Using the above inequality and (H2), direct calculations show that

$$
\left|\tilde{b}-\tilde{b}_{\delta}\right| \leq 2|\tilde{b}|+\delta|\tilde{\rho}|=2 b_{1}+\delta|\tilde{\rho}| \leq 2 \delta a_{1}+\delta|\tilde{\rho}|<2 \delta \bar{\sigma} .
$$

Then by (H2), it is easy to see that

$$
\begin{aligned}
\left|F_{1}^{ \pm}\left(t^{n} h ; \tilde{a}, \tilde{b}\right)\right| & \leq C\|h\|_{L^{\infty}([1, \infty))} \int_{1}^{\infty} \frac{t^{n}}{\sqrt{t^{2}-1}} e^{\left|\left(\tilde{b}-\tilde{b}_{\delta}\right) \mu_{ \pm}(t)\right|-k t \operatorname{Im} \tilde{\rho}_{\delta}} \mathrm{d} t \\
& \leq C\|h\|_{L^{\infty}([1, \infty))} \int_{1}^{\infty} \frac{t^{n}}{\sqrt{t^{2}-1}} e^{\left(10 \delta \gamma_{0}^{-1}-\sqrt{1+\delta^{2}} \gamma_{0}\right) k t \bar{\sigma}} \mathrm{d} t \\
& \leq C\|h\|_{L^{\infty}([1, \infty))} e^{-\frac{1}{2} k \gamma_{0} \bar{\sigma}+1} \int_{1}^{\infty} \frac{t^{n} e^{-t}}{\sqrt{t^{2}-1}} \mathrm{~d} t \\
& \leq C\|h\|_{L^{\infty}([1, \infty))} e^{-\frac{1}{2} k \gamma_{0} \bar{\sigma}} .
\end{aligned}
$$

Then the proof is completed. 
Remark 4.2. Define $\tilde{b}^{\prime}=\left[\left(\tilde{x}_{2}-y_{2}\right)^{2}\right]^{1 / 2}$. Using similar arguments as in (4.6), we find that

$$
\operatorname{Im} r(\tilde{\boldsymbol{x}}, \boldsymbol{y})=\operatorname{Im}\left[\left(\tilde{x}_{1}-y_{1}\right)^{2}+\left(\tilde{x}_{2}-y_{2}\right)^{2}\right]^{1 / 2} \geq \gamma_{0} \bar{\sigma} .
$$

Then Lemma 4.3 also holds by replacing $\tilde{b}$ with $\tilde{b}^{\prime}$.

The following lemmas on the estimate of the modified Green function $\tilde{G}$ will play an important role in the following analysis.

Lemma 4.4. Let $\tilde{a}, \tilde{b}, \tilde{\rho}, \tilde{b}_{\delta}$ and $\tilde{\rho}_{\delta}$ be defined in (4.5) and (4.8) for any $x \in \Gamma_{2}, y \in \bar{\Omega}_{1}$. Assume $b_{1}>\delta a_{1}$. Let (H1)-(H2) be satisfied. Then

$$
|\tilde{G}(\boldsymbol{x}, \boldsymbol{y})| \leq C[1+\bar{\sigma}(d+\bar{\sigma})] e^{-\gamma_{0} k \bar{\sigma}}+\frac{Z e^{-Z\left(L_{2}+d_{2}\right)}}{\sqrt{Z^{2}+k^{2}}}, \quad \forall \boldsymbol{x} \in \Gamma_{2}, \quad \boldsymbol{y} \in \bar{\Omega}_{1} .
$$

Proof. By (2.5) and the method of Cagniard-de Hoop transform (cf. [19]) we know the Green function $\Phi(\boldsymbol{x}, \boldsymbol{y})=\frac{\mathbf{i}}{4} H_{0}^{(1)}(k|x-y|)$ satisfies

$$
\Phi(x, y)=\frac{1}{2 \pi} \int_{1}^{\infty} \frac{1}{\sqrt{t^{2}-1}} e^{\mathrm{i} k|x-y| t} \mathrm{~d} t
$$

Thus by (2.6) and Lemma 4.3, we know that,

$$
\tilde{G}(\boldsymbol{x}, \boldsymbol{y})=\frac{1}{2} F(1 ; \tilde{a}, \tilde{b})-\frac{1}{2} F\left(1 ; \tilde{a}, \tilde{b}^{\prime}\right)+F(f ; \tilde{a}, \tilde{b})-S(\tilde{x}, \boldsymbol{y}),
$$

where $S$ is defined in $(2.7), \tilde{b}^{\prime}=\left[\left(\tilde{x}_{2}-y_{2}\right)^{2}\right]^{1 / 2}$ and

$$
f(t)=-\mathbf{i}\left(\frac{\mu_{+}(t)}{Z+\mathbf{i} \mu_{+}(t)}+\frac{\mu_{-}(t)}{Z+\mathbf{i} \mu_{-}(t)}\right) .
$$

In Lemma A.3, we have proved that

$$
|f(t)| \leq 1+C \bar{\sigma}(d+\bar{\sigma}) .
$$

Then from Lemma 4.3, there exists a constant $C$ such that

$$
|F(f ; \tilde{a}, \tilde{b})| \leq C[1+\bar{\sigma}(d+\bar{\sigma})] e^{-k \gamma_{0} \bar{\sigma}} .
$$

An application of Lemma 4.3 and Remark 4.2 shows that

$$
|F(1 ; \tilde{a}, \tilde{b})|+\left|F\left(1 ; \tilde{a}, \tilde{b}^{\prime}\right)\right| \leq C e^{-k \gamma_{0} \bar{\sigma}} .
$$

By (2.7) and Assumption (H1), the term of surface waves satisfies

$$
|S(\tilde{x}, y)|=\frac{Z e^{-Z\left(x_{2}+y_{2}\right)} e^{-\sqrt{Z^{2}+k^{2}} \operatorname{Im}(\tilde{a})}}{\sqrt{Z^{2}+k^{2}}} \leq \frac{Z \max \left(e^{-Z\left(L_{2}+d_{2}\right)}, e^{-\sqrt{Z^{2}+k^{2}} \bar{\sigma}}\right)}{\sqrt{Z^{2}+k^{2}}} .
$$

The proof is completed by substituting (4.20), (4.21) and (4.22) into (4.17). 
Lemma 4.5. Let $\tilde{a}, \tilde{b}, \tilde{\rho}, \tilde{b}_{\delta}$ and $\tilde{\rho}_{\delta}$ be defined in (4.5) and (4.8) for any $\boldsymbol{x} \in \Gamma_{2}, \boldsymbol{y} \in \bar{\Omega}_{1}$. Assume $b_{1} \leq \delta a_{1}$. Let (H1)-(H2) be satisfied. Then

$$
|\tilde{G}(\boldsymbol{x}, \boldsymbol{y})| \leq C[1+(d+\bar{\sigma})] e^{-\frac{1}{2} \gamma_{0} k \bar{\sigma}}, \quad \forall x \in \Gamma_{2}, \quad \boldsymbol{y} \in \bar{\Omega}_{1} .
$$

Proof. Let

$$
\begin{aligned}
& I_{1}(h ; \tilde{a}, \tilde{b})=\frac{1}{2 \pi} \int_{1}^{\infty} h\left(\xi_{+}(t)\right) \Lambda_{+}(t) e^{\mathbf{i}\left(\tilde{b}-\tilde{b}_{\delta}\right) \Lambda_{+}(t)} \frac{e^{\mathbf{i} k \tilde{\rho}_{\delta} t}}{\sqrt{t^{2}-1}} \mathrm{~d} t \\
& I_{2}(h ; \tilde{a}, \tilde{b})=\frac{1}{2 \pi} \int_{1}^{\sqrt{2}} h\left(\xi_{-}(t)\right) \Lambda_{-}(t) e^{\mathbf{i}\left(\tilde{b}-\tilde{b}_{\delta}\right) \Lambda_{-}(t)} \frac{e^{\mathbf{i} k \tilde{\rho}_{\delta} t}}{\sqrt{t^{2}-1}} \mathrm{~d} t \\
& I_{3}(h ; \tilde{a}, \tilde{b})=\frac{1}{2 \pi} k e^{\mathbf{i} \sqrt{2} k \tilde{\rho}_{\delta}} \int_{0}^{\infty} h(\hat{\zeta}(t)) e^{\left.\mathbf{i}\left[\tilde{b} \mu(\hat{\zeta}(t))-\tilde{b}_{\delta} \Lambda_{-}(\sqrt{2})\right)\right]} e^{\mathbf{i} k \tilde{a} t} \mathrm{~d} t
\end{aligned}
$$

where $\xi_{ \pm}(t)$ are defined in (4.11) and $\hat{\xi}(t):=k t+\xi_{0}$ with

$$
\xi_{0}=\xi_{-}(\sqrt{2})=\frac{k}{\tilde{\rho}_{\delta}}\left(\sqrt{2} \tilde{a}-\mathbf{i} \tilde{b}_{\delta}\right) .
$$

Thus by (2.6) and Lemma 4.3, we know that

$$
\tilde{G}(\boldsymbol{x}, \boldsymbol{y})=\sum_{i=1}^{3}\left(\frac{1}{2} I_{i}(1 / \mu ; \tilde{a}, \tilde{b})-\frac{1}{2} I_{i}\left(1 / \mu ; \tilde{a}, \tilde{b}^{\prime}\right)+I_{i}(f ; \tilde{a}, \tilde{b})\right)-S(\tilde{x}, \boldsymbol{y}),
$$

where $S$ is defined in $(2.7), \tilde{b}^{\prime}=\left[\left(\tilde{x}_{2}-y_{2}\right)^{2}\right]^{1 / 2}$ and

$$
f=\frac{-\mathbf{i}}{Z+\mathbf{i} \mu} \quad \text { with } \mu=\mu\left(\xi_{+}\right) \text {in } I_{1}, \quad \mu=\mu\left(\xi_{-}\right) \quad \text { in } I_{2}, \quad \mu=\mu(\hat{\xi}) \quad \text { in } I_{3} \text {. }
$$

Since $b_{1} \leq \delta a_{1}$, then $\operatorname{Im} \tilde{a}=\bar{\sigma}$, the term of surface waves satisfies

$$
|S(\tilde{\boldsymbol{x}}, \boldsymbol{y})|=\frac{Z e^{-Z\left(x_{2}+y_{2}\right)} e^{-\sqrt{Z^{2}+k^{2}} \operatorname{Im} \tilde{a}}}{\sqrt{Z^{2}+k^{2}}} \leq \frac{Z e^{-\sqrt{Z^{2}+k^{2}} \bar{\sigma}}}{\sqrt{Z^{2}+k^{2}}} .
$$

By arguments similar to those in Lemma 4.3, we obtain for any $h \in L^{\infty}([1, \infty))$,

$$
\left|I_{2}(h ; \tilde{a}, \tilde{b})\right| \leq C\|\mu h\|_{L^{\infty}([1, \infty))} e^{-\frac{1}{2} k \gamma_{0} \bar{\sigma}} .
$$

An application of Lemma 4.3, Remark 4.2 and (4.26) shows that

$$
\begin{aligned}
& \sum_{i=1}^{2}\left|I_{i}(1 / \mu ; \tilde{a}, \tilde{b})\right|=\left|F_{1}^{+}(1 ; \tilde{a}, \tilde{b})\right|+\left|I_{2}(1 / \mu ; \tilde{a}, \tilde{b})\right| \leq C e^{-\frac{1}{2} k \gamma_{0} \bar{\sigma}} \\
& \sum_{i=1}^{2}\left|I_{i}\left(1 / \mu ; \tilde{a}, \tilde{b}^{\prime}\right)\right|=\left|F_{1}^{+}\left(1 ; \tilde{a}, \tilde{b}^{\prime}\right)\right|+\left|I_{2}\left(1 / \mu ; \tilde{a}, \tilde{b}^{\prime}\right)\right| \leq C e^{-\frac{1}{2} k \gamma_{0} \bar{\sigma}}
\end{aligned}
$$


By (4.24) and Lemma A.4, we have

$$
|\mu f|=\left|\frac{-\mathbf{i} \mu}{Z+\mathbf{i} \mu}\right| \leq 1+\left|\frac{Z}{Z+\mathbf{i} \mu}\right| \leq 1+C(d+\bar{\sigma}) .
$$

Then from Lemma 4.3 and (4.26),

$$
\sum_{i=1}^{2}\left|I_{i}(f ; \tilde{a}, \tilde{b})\right|=\left|F_{1}^{+}(\mu f ; \tilde{a}, \tilde{b})\right|+\left|I_{2}(f ; \tilde{a}, \tilde{b})\right| \leq C[1+(d+\bar{\sigma})] e^{-\frac{1}{2} \gamma_{0} k \bar{\sigma}}
$$

Now we estimate $I_{3}$. By Lemma A.2, $\operatorname{Im} \hat{\xi}(t)=\operatorname{Im} \xi_{-}(\sqrt{2})<0$ for all $t \geq 0$. Then the convention in (2.2) shows that

$$
\operatorname{Re} \mu(\hat{\xi}(t)) \geq 0, \quad \operatorname{sign}[\operatorname{Im} \mu(\hat{\zeta}(t))]=-\operatorname{sign}\left[\operatorname{Im} \hat{\xi}^{2}(t)\right]=-\operatorname{sign}[\operatorname{Im} \hat{\xi}(t)]>0,
$$

which implies that $\tilde{b} \mu(\hat{\xi})$ has nonnegative imaginary part. Then similar argument as in Lemma 4.3 yields

$$
\left|e^{\left.\mathbf{i}\left[\tilde{b} \mu(\hat{\xi})-\tilde{b}_{\delta} \Lambda_{-}(\sqrt{2})\right)\right]}\right| \leq\left|e^{-\mathbf{i} \tilde{b}_{\delta} \Lambda_{-}(\sqrt{2})}\right| \leq e^{\left|\tilde{b}_{\delta}\right| \mid \Lambda_{-}(\sqrt{2})} \mid \leq e^{2 \delta \bar{\sigma} \cdot 5 \sqrt{2} k \gamma_{0}^{-1}} .
$$

By Lemma A.5, we have proved that

$$
\left|\frac{1}{\mu(\hat{\zeta}(t))}\right| \leq C, \quad|f(t)|=\left|\frac{-\mathbf{i}}{Z+\mathbf{i} \mu(\hat{\zeta}(t))}\right| \leq C .
$$

Then from (4.30), (4.31) and the assumption that $\delta<\frac{\gamma_{0}^{2}}{20}$, we have

$$
\begin{aligned}
& \left|I_{3}(1 / \mu ; \tilde{a}, \tilde{b})-I_{3}\left(1 / \mu ; \tilde{a}, \tilde{b}^{\prime}\right)+I_{3}(f ; \tilde{a}, \tilde{b})\right| \\
\leq & C e^{-\sqrt{2} \gamma_{0} k \bar{\sigma}} \int_{0}^{\infty} e^{10 \sqrt{2} \delta \bar{\sigma} k \gamma_{0}^{-1}} e^{-k \bar{\sigma} t} \mathrm{~d} t \leq C e^{-\sqrt{2} \gamma_{0} k \bar{\sigma}} .
\end{aligned}
$$

The proof is completed by inserting (4.25), (4.27)-(4.29) and (4.32) into (4.23).

Lemma 4.6. Let (H1)-(H2) be satisfied and let $m, n \geq 0$ be integers. Then for $\boldsymbol{x}=\left(x_{1}, x_{2}\right) \in \Gamma_{2}$, $\boldsymbol{y}=\left(y_{1}, y_{2}\right) \in \bar{\Omega}_{1}$, if $b_{1}>\delta a_{1}$, for $1 \leq i, j \leq 2$,

$$
\left|\frac{\partial^{m+n}}{\partial x_{i}^{m} \partial y_{j}^{n}} \tilde{G}(\boldsymbol{x}, \boldsymbol{y})\right| \leq \alpha_{0}^{m}\left(C[1+\bar{\sigma}(d+\bar{\sigma})] e^{-\gamma_{0} k \bar{\sigma}}+\left(\sqrt{Z^{2}+k^{2}}\right)^{m+n} \frac{Z e^{-Z\left(L_{2}+d_{2}\right)}}{\sqrt{Z^{2}+k^{2}}}\right),
$$

and if $b_{1} \leq \delta a_{1}$, for $1 \leq i, j \leq 2$,

$$
\left|\frac{\partial^{m+n}}{\partial x_{i}^{m} \partial y_{j}^{n}} \tilde{G}(x, y)\right| \leq C \alpha_{0}^{m}[1+(d+\bar{\sigma})] e^{-\frac{1}{2} \gamma_{0} k \bar{\sigma}},
$$

where $\alpha_{0}=\max _{x \in \Gamma_{2}}\left(\left|\alpha_{1}\left(x_{1}\right)\right|,\left|\alpha_{2}\left(x_{2}\right)\right|\right)$. 
Proof. For the surface wave $S(\tilde{\boldsymbol{x}}, \boldsymbol{y})$. By (2.7), it is easy to see that if $b_{1}>\delta a_{1}$,

$$
\left|\frac{\partial^{m+n}}{\partial x_{i}^{m} \partial y_{j}^{n}} \tilde{S}(\boldsymbol{x}, \boldsymbol{y})\right| \leq \alpha_{0}^{m}\left(\sqrt{Z^{2}+k^{2}}\right)^{m+n} \frac{Z e^{-Z\left(L_{2}+d_{2}\right)}}{\sqrt{Z^{2}+k^{2}}},
$$

and if $b_{1} \leq \delta a_{1}$,

$$
\left|\frac{\partial^{m+n}}{\partial x_{i}^{m} \partial y_{j}^{n}} \tilde{S}(\boldsymbol{x}, \boldsymbol{y})\right| \leq \alpha_{0}^{m}\left(\sqrt{Z^{2}+k^{2}}\right)^{m+n} \frac{Z e^{-\sqrt{Z^{2}+k^{2}} \bar{\sigma}}}{\sqrt{Z^{2}+k^{2}}} .
$$

For the propagating waves, we only consider the case of $b_{1} \leq \delta a_{1}$. The proof for the case of $b_{1}>\delta a_{1}$ is similar and simpler. By direct calculations, we have

$$
\left|\frac{\partial^{m+n} \mu\left(\xi_{ \pm}(t)\right)}{\partial \tilde{a}^{m} \partial \tilde{b}^{n}}\right| \leq C \frac{k t}{|\tilde{\rho}|^{m+n}}, \quad\left|\frac{\partial^{m+n} \mu(\hat{\zeta}(t))}{\partial \tilde{a}^{m} \partial \tilde{b}^{n}}\right| \leq C \frac{k}{|\tilde{\rho}|^{m+n}}, \quad \forall m, n \geq 0,
$$

where $\xi_{ \pm}(t)$ are defined in $(4.11)$ and $\hat{\xi}(t):=k t+\xi_{0}$ with $\xi_{0}=\xi_{-}(\sqrt{2})=\frac{k}{\tilde{\rho}_{\delta}}\left(\sqrt{2} \tilde{a}-\mathbf{i} \tilde{b}_{\delta}\right)$.

By (4.33) and elaborate calculations,

$$
\begin{aligned}
& \left|\frac{\partial^{m+n}}{\partial \tilde{a}^{m} \partial \tilde{b}^{n}}\left(f\left(\tilde{\zeta}_{ \pm}(t)\right) e^{\mathbf{i}\left(\tilde{b}-\tilde{b}_{\delta}\right) \Lambda_{ \pm}(t)}\right)\right| \leq C k t\left|\tilde{\rho}_{\delta}\right| \cdot\left|e^{\mathbf{i}\left(\tilde{b}-\tilde{b}_{\delta}\right) \Lambda_{ \pm}(t)}\right|, \\
& \left|\frac{\partial^{m+n}}{\partial \tilde{a}^{m} \partial \tilde{b}^{n}}\left(f(\hat{\zeta}(t)) e^{\left.\mathbf{i}\left[\tilde{b} \mu(\hat{\xi}(t))-\tilde{b}_{\delta} \Lambda_{-}(\sqrt{2})\right)\right]}\right)\right| \leq C k\left|\tilde{\rho}_{\delta}\right| \cdot\left|e^{\left.\mathbf{i}\left[\tilde{b} \mu(\hat{\xi}(t))-\tilde{b}_{\delta} \Lambda_{-}(\sqrt{2})\right)\right]}\right|,
\end{aligned}
$$

where $f(\xi)=-\mathbf{i} /(Z+\mathbf{i} \mu)$.

Consider the representation of $\tilde{G}(\boldsymbol{x}, \boldsymbol{y})$ given by (4.23). By arguments similar to the proof of Lemma 4.3, we obtain

$$
\left|\frac{\partial^{m+n} I_{l}(f ; \tilde{a}, \tilde{b})}{\partial \tilde{a}^{m} \partial \tilde{b}^{n}}\right| \leq C(d+\bar{\sigma}) e^{-\frac{1}{2} k \gamma_{0} \bar{\sigma}}, \quad l=1,2,3 .
$$

Similarly, we derive that

$$
\left|\frac{\partial^{m+n} I_{l}(1 / \mu ; \tilde{a}, \tilde{b})}{\partial \tilde{a}^{m} \partial \tilde{b}^{n}}\right| \leq C e^{-\frac{1}{2} k \gamma_{0} \bar{\sigma}}, \quad l=1,2,3 .
$$

Then by the chain rule,

$$
\left|\frac{\partial f}{\partial x_{1}}\right|=\left|\frac{\partial f}{\partial \tilde{a}} \frac{\partial \tilde{a}}{\partial x_{1}}\right| \leq \alpha_{0} \frac{\partial f}{\partial \tilde{a}^{\prime}}, \quad\left|\frac{\partial f}{\partial x_{2}}\right|=\left|\frac{\partial f}{\partial \tilde{b}} \frac{\partial \tilde{b}}{\partial x_{2}}\right| \leq \alpha_{0} \frac{\partial f}{\partial \tilde{b}^{\prime}},
$$

and

$$
\left|\frac{\partial f}{\partial y_{1}}\right|=\left|\frac{\partial f}{\partial \tilde{a}} \frac{\partial \tilde{a}}{\partial y_{1}}\right| \leq \frac{\partial f}{\partial \tilde{a}^{\prime}} \quad\left|\frac{\partial f}{\partial y_{2}}\right|=\left|\frac{\partial f}{\partial \tilde{b}} \frac{\partial \tilde{b}}{\partial y_{2}}\right| \leq \frac{\partial f}{\partial \tilde{b}} .
$$

the proof is completed. 
Note that $\bar{\sigma} e^{-\gamma_{0} k \bar{\sigma}} \leq C e^{-\frac{1}{2} \gamma_{0} k \bar{\sigma}}$ for some constant $C$. To simplify the notation, we use

$$
\left|\frac{\partial^{m+n}}{\partial x_{i}^{m} \partial y_{j}^{n}} \tilde{G}(x, y)\right| \leq \alpha_{0}^{m}\left(C[1+(d+\bar{\sigma})] e^{-\frac{1}{2} \gamma_{0} k \bar{\sigma}}+\left(\sqrt{Z^{2}+k^{2}}\right)^{m+n} \frac{Z e^{-Z\left(L_{2}+d_{2}\right)}}{\sqrt{Z^{2}+k^{2}}}\right)
$$

for both cases of $b_{1}>\delta a_{1}$ and $b_{1} \leq \delta a_{1}$ in the rest of this paper.

\subsection{Estimation of the PML wave propagation operator}

Let $\Gamma$ be any one-dimensional Lipschitz manifold. Throughout the paper we shall use the following weighted norm $\|\cdot\|_{H^{\frac{1}{2}}(\Gamma)}$

$$
\|v\|_{H^{\frac{1}{2}(\Gamma)}}^{2}=|\Gamma|^{-1}\|v\|_{L^{2}(\Gamma)}^{2}+|v|_{\frac{1}{2}, \Gamma^{\prime}}^{2}, \quad|v|_{\frac{1}{2}, \Gamma}^{2}=\int_{\Gamma} \int_{\Gamma} \frac{|v(x)-v(y)|^{2}}{|x-y|^{2}} \mathrm{~d} S_{x} \mathrm{~d} S_{y} .
$$

Lemma 4.7. Let (H1)-(H2) be satisfied. Then for any $f \in H^{\frac{1}{2}}\left(\Gamma_{1}\right)$,

$$
\left\|\widetilde{\Psi}_{\mathrm{DL}}(f)\right\|_{H^{\frac{1}{2}\left(\Gamma_{2}\right)}} \leq \alpha_{0}\left(C \zeta_{p} e^{-\frac{1}{2} \gamma_{0} k \bar{\sigma}}+\zeta_{s} e^{-Z\left(L_{2}+d_{2}\right)}\right)\|f\|_{H^{\frac{1}{2}}\left(\Gamma_{1}\right)^{\prime}}
$$

where $\zeta_{p}=1+(d+\bar{\sigma})$ and $\zeta_{s}=1+Z$.

Proof. For convenience we denote $v=\widetilde{\Psi}_{\mathrm{DL}}(f)$. Then from (4.2) and Lemma 4.6 we know that, for any $x \in \Gamma_{2}$,

$$
\begin{aligned}
& |v(x)| \leq|\widetilde{G}(x, \cdot)|_{W^{1, \infty}\left(\Gamma_{1}\right)}\|f\|_{L^{1}\left(\Gamma_{1}\right)} \leq \alpha_{0}\left(C \zeta_{p} e^{-\frac{1}{2} \gamma_{0} k \bar{\sigma}}+\frac{Z e^{-Z\left(L_{2}+d_{2}\right)}}{\sqrt{Z^{2}+k^{2}}}\right)\|f\|_{L^{1}\left(\Gamma_{1}\right)}, \\
& |\nabla v(x)| \leq\left|\nabla_{x} \widetilde{G}(x, \cdot)\right|_{W^{1, \infty}\left(\Gamma_{1}\right)}\|f\|_{L^{1}\left(\Gamma_{1}\right)} \leq \alpha_{0}\left(C \zeta_{p} e^{-\frac{1}{2} \gamma_{0} k \bar{\sigma}}+Z e^{-Z\left(L_{2}+d_{2}\right)}\right)\|f\|_{L^{1}\left(\Gamma_{1}\right)} .
\end{aligned}
$$

From (4.34), we deduce that

$$
\|v\|_{H^{\frac{1}{2}\left(\Gamma_{2}\right)}} \leq\|v\|_{L^{\infty}\left(\Gamma_{2}\right)}+\left|\Gamma_{2}\right|\|\nabla v\|_{L^{\infty}\left(\Gamma_{2}\right)} \leq \varepsilon\|f\|_{L^{1}\left(\Gamma_{1}\right)} \leq \varepsilon\|f\|_{H^{\frac{1}{2}\left(\Gamma_{1}\right)^{\prime}}}
$$

where

$$
\varepsilon=\alpha_{0}\left(C \zeta_{p} e^{-\frac{1}{2} \gamma_{0} k \bar{\sigma}}+(1+Z) e^{-Z\left(L_{2}+d_{2}\right)}\right) .
$$

This completes the proof.

Lemma 4.8. Let (H1)-(H2) be satisfied. Then for any $\lambda \in H^{-\frac{1}{2}}\left(\Gamma_{1}\right)$,

$$
\left\|\widetilde{\Psi}_{\mathrm{SL}}(\lambda)\right\|_{H^{\frac{1}{2}\left(\Gamma_{2}\right)}} \leq \alpha_{0}\left(C \zeta_{p} e^{-\frac{1}{2} \gamma_{0} k \bar{\sigma}}+\zeta_{S} e^{-Z\left(L_{2}+d_{2}\right)}\right)\|\lambda\|_{H^{-\frac{1}{2}\left(\Gamma_{1}\right)}} .
$$


Proof. For convenience we denote $v=\widetilde{\Psi}_{\mathrm{SL}}(\lambda)$. Then from (4.2) and Lemma 4.6 we know that, for any $x \in \Gamma_{2}$,

$$
\begin{aligned}
|v(x)| & \leq\|\widetilde{G}(x, \cdot)\|_{H^{1 / 2}\left(\Gamma_{1}\right)}\|\lambda\|_{H^{-1 / 2}\left(\Gamma_{1}\right)} \\
\leq & C\left(\|\widetilde{G}(x, \cdot)\|_{L^{\infty}\left(\Gamma_{1}\right)}+\left|\Gamma_{1}\right||\widetilde{G}(x, \cdot)|_{W^{1, \infty}\left(\Gamma_{1}\right)}\right)\|\lambda\|_{H^{-1 / 2}\left(\Gamma_{1}\right)}, \\
|\nabla v(x)| & \leq\left\|\nabla_{x} \widetilde{G}(x, \cdot)\right\|_{H^{1 / 2}\left(\Gamma_{1}\right)}\|\lambda\|_{H^{-1 / 2}\left(\Gamma_{1}\right)} \\
& \leq C\left(\left\|\nabla_{x} \widetilde{G}(x, \cdot)\right\|_{L^{\infty}\left(\Gamma_{1}\right)}+\left|\Gamma_{1}\right|\left|\nabla_{x} \widetilde{G}(x, \cdot)\right|_{W^{1, \infty}\left(\Gamma_{1}\right)}\right)\|\lambda\|_{H^{-1 / 2}\left(\Gamma_{1}\right)} .
\end{aligned}
$$

We complete the proof by using Lemmas 4.5-4.6 and similar arguments as in the proof of Lemma 4.7.

Theorem 4.1. Let (H1)-(H2) be satisfied. Then for any $f \in H^{\frac{1}{2}}\left(\Gamma_{1}\right)$,

$$
\|\mathbb{E}(f)\|_{H^{\frac{1}{2}\left(\Gamma_{2}\right)}} \leq \alpha_{0}\left(C \zeta_{p} e^{-\frac{1}{2} \gamma_{0} k \bar{\sigma}}+\zeta_{s} e^{-Z\left(L_{2}+d_{2}\right)}\right)\|f\|_{H^{\frac{1}{2}}\left(\Gamma_{1}\right)} .
$$

Proof. This theorem is a direct consequence of Lemmas $4.7-4.8$ and the continuity of the DtN operator $T: H^{1 / 2}\left(\Gamma_{1}\right) \mapsto H^{-1 / 2}\left(\Gamma_{1}\right)$ which is defined by using the scattering problem (3.7).

Remark 4.3. Theorem 4.1 implies that the solution $u$ of problem (1.1) satisfies

$$
\|\tilde{u}\|_{H^{\frac{1}{2}\left(\Gamma_{2}\right)}}=\|\mathbb{E}(u)\|_{H^{\frac{1}{2}\left(\Gamma_{2}\right)}} \leq \alpha_{0}\left(C \zeta_{p} e^{-\frac{1}{2} \gamma_{0} k \bar{\sigma}}+\zeta_{s} e^{-Z\left(L_{2}+d_{2}\right)}\right)\|u\|_{H^{\frac{1}{2}}\left(\Gamma_{1}\right)} .
$$

Thus $\tilde{u}$ decays exponentially in the PML as we enlarge the thickness of the layer.

Heuristically, from (3.6) we know that, inside the PML, the propagating waves $u_{p}$ decays at the rate $\alpha_{0} \zeta_{p} e^{-\frac{1}{2} \gamma_{0} k \bar{\sigma}}$, the surface waves $u_{s}$ decays at the rate $\alpha_{0} \zeta_{p} e^{-\frac{1}{2} \gamma_{0} k \bar{\sigma}}$ in the horizontal direction but at the rate $\alpha_{0} \zeta_{s} e^{-Z\left(L_{2}+d_{2}\right)}$ in the vertical direction.

\section{Exponential convergence of the UPML method}

Since $\tilde{u}$ decays exponentially in the PML, we define the approximation problem by setting homogeneous Dirichlet boundary condition on the outer boundary:

$$
\begin{array}{ll}
\nabla \cdot(A \nabla \hat{u})+\alpha_{1} \alpha_{2} k^{2} \hat{u}=0 & \text { in } \Omega_{2}, \\
\frac{\partial \hat{u}}{\partial n}-\mathbf{i} k \beta \hat{u}=g & \text { on } \Sigma_{2}, \\
\hat{u}=0 & \text { on } \Gamma_{2},
\end{array}
$$


where we extend $g$ by zero to $\Sigma_{\infty}$. The well-posedness of the PML problem (5.1) and the convergence of the solution $\hat{u}$ to the solution of the original scattering problem will be postponed to the end of this section.

A weak formulation of (5.1) reads: Find $\hat{u} \in H^{1}\left(\Omega_{1}\right)$ such that

$$
\hat{a}(\hat{u}, v)=\langle g, v\rangle_{\Sigma_{p}}, \quad \forall v \in H^{1}\left(\Omega_{1}\right),
$$

where the bilinear form $\hat{a}$ : $H^{1}\left(\Omega_{1}\right) \times H^{1}\left(\Omega_{1}\right) \mapsto \mathbb{C}$ is defined by

$$
\hat{a}(v, w)=\int_{\Omega_{1}}\left(\nabla v \cdot \nabla \bar{w}-k^{2} v \bar{w}\right) \mathrm{d} x-\langle\hat{T} v, \bar{w}\rangle_{\Gamma_{1}}-\mathbf{i} k\langle\beta v, \bar{w}\rangle_{\Sigma_{1}}
$$

The DtN operator $\hat{T}: H^{1 / 2}\left(\Gamma_{1}\right) \mapsto H^{-1 / 2}\left(\Gamma_{1}\right)$ is defined by $\hat{T} f:=\frac{\partial \hat{w}}{\partial n}$ for any $f \in H^{1 / 2}\left(\Gamma_{1}\right)$, where $\hat{w}$ solves

$$
\begin{array}{ll}
\nabla \cdot(A \nabla \hat{w})+\alpha_{1} \alpha_{2} k^{2} \hat{w}=0 & \text { in } \Omega_{\mathrm{PML}}:=\Omega_{2} \backslash \bar{\Omega}_{1}, \\
\frac{\partial \hat{w}}{\partial x_{2}}+Z \hat{w}=0 & \text { on } \Sigma_{\mathrm{PML}}:=\Sigma_{\infty} \cap \partial \Omega_{\mathrm{PML}}, \\
\hat{w}=f \quad \text { on } \Gamma_{1}, & \hat{w}=0 \quad \text { on } \Gamma_{2} .
\end{array}
$$

The original problem (3.13) and the PML problem (5.2) only differ from the DtN operators $T, \hat{T}$. Now we shall study the well-posedness of (5.4) and estimate $T-\hat{T}$.

Remark 5.1. The boundary conditions in (5.1b)-(5.1c) are compatible at the two points $\boldsymbol{p}_{ \pm}= \pm\left(L_{1}+d_{1}, 0\right)$. In fact, we have

$$
-\lim _{\substack{x \in \Sigma_{2} \\ x \rightarrow p_{ \pm}}}\left(\frac{\partial \hat{u}}{\partial n}-\mathbf{i} k \beta \hat{u}\right)(x)=\lim _{\substack{x \in \Sigma_{2} \\ x \rightarrow p_{ \pm}}}\left(\frac{\partial \hat{u}}{\partial x_{2}}+Z \hat{u}\right)(x)=0=\lim _{\substack{x \in \Gamma_{2} \\ x \rightarrow p_{ \pm}}}\left(\frac{\partial \hat{u}}{\partial x_{2}}+Z \hat{u}\right)(x),
$$

where $\hat{u}$ is assumed to be piecewise smooth such that the above limits make sense.

\subsection{The PML equation in the layer}

From (3.7), (4.3) and (5.4), for any $f \in H^{1 / 2}\left(\Gamma_{1}\right),(T-\hat{T}) f=\frac{\partial w}{\partial n}$, where $w$ solves the boundary value problem of the PML equation in the layer:

$$
\begin{array}{ll}
\nabla \cdot(A \nabla w)+\alpha_{1} \alpha_{2} k^{2} w=0 & \text { in } \Omega_{\mathrm{PML},} \\
\frac{\partial w}{\partial x_{2}}+Z w=0 & \text { on } \Sigma_{\mathrm{PML}}, \\
w=0 \quad \text { on } \Gamma_{1}, & w=\mathbb{E}(f) \text { on } \Gamma_{2} .
\end{array}
$$

Introduce the bilinear form $c: H^{1}\left(\Omega_{\mathrm{PML}}\right) \times H^{1}\left(\Omega_{\mathrm{PML}}\right) \rightarrow \mathbb{C}$ as follows

$$
c(\phi, \varphi)=\int_{\Omega_{\mathrm{PML}}}\left(A \nabla \phi \cdot \nabla \bar{\varphi}-\alpha_{1} \alpha_{2} k^{2} \phi \bar{\varphi}\right) \mathrm{d} x-Z\left\langle\alpha_{1} \phi, \bar{\varphi}\right\rangle_{\Sigma_{\mathrm{PML}}} \quad \forall \phi, \varphi \in H^{1}\left(\Omega_{\mathrm{PML}}\right) .
$$


Then a weak formulation of (5.5) reads: Find $w \in H^{1}\left(\Omega_{\mathrm{PML}}\right)$ such that $w=0$ on $\Gamma_{1}, w=\mathbb{E}(f)$ on $\Gamma_{2}$ and

$$
c(w, \phi)=0, \quad \forall \phi \in H_{\Gamma_{1} \cup \Gamma_{2}}^{1}\left(\Omega_{\mathrm{PML}}\right),
$$

where $H_{\Gamma_{1} \cup \Gamma_{2}}^{1}\left(\Omega_{\mathrm{PML}}\right)$ is defined similarly as in (3.11).

In the reminder of this paper we make the assumption on the medium property:

(H3) $\quad \sigma_{j}(t) \equiv \sigma \geq 1, \forall|t| \geq L_{j}, j=1,2$, where $\sigma$ is a constant.

This assumption is not restrictive in practical applications and allows us to prove the coercivity of the bilinear form $c$. The assumption $\sigma \geq 1$ is just used to simplify the constants in the convergence analysis. The following lemma shows that (5.4), (5.5) have unique solutions and the DtN operator $\hat{T}$ is well-defined.

Throughout the paper we will use the weighted $H^{1}$-norm for any $\Omega \subset \mathbb{R}^{2}$ :

$$
\|\mid \varphi\|_{H^{1}(\Omega)}=\left(\|\nabla \varphi\|_{L^{2}(\Omega)}^{2}+\|k \varphi\|_{L^{2}(\Omega)}^{2}\right)^{1 / 2} .
$$

Lemma 5.1. Let (H3) be satisfied. Then (5.6) has a unique solution and the bilinear form $c(\cdot, \cdot)$ satisfies, for any $\varphi \in H_{\Gamma_{1} \cup \Gamma_{2}}^{1}\left(\Omega_{\mathrm{PML}}\right)$,

$$
|c(\varphi, \varphi)| \geq \frac{\hat{C}_{\mathrm{IS}}}{24}\|\varphi\|_{H^{1}\left(\Omega_{\mathrm{PML}}\right)}^{2}, \quad \hat{C}_{\mathrm{IS}}^{-1}=\sigma^{2}\left(1+Z d+k^{2} d^{2}\right) .
$$

Proof. It is clear that $\bar{\Omega}_{\mathrm{PML}}=\bar{\Omega}_{c} \cup \bar{\Omega}_{1} \cup \bar{\Omega}_{2}$, where

$$
\begin{aligned}
& \Omega_{c}=\left\{x \in \Omega_{\mathrm{PML}}:\left|x_{1}\right|>L_{1}, x_{2}>L_{2}\right\}, \\
& \Omega_{1}=\left\{x \in \Omega_{\mathrm{PML}}:\left|x_{1}\right|>L_{1}, x_{2}<L_{2}\right\}, \\
& \Omega_{2}=\left\{x \in \Omega_{\mathrm{PML}}:\left|x_{1}\right|<L_{1}, x_{2}>L_{2}\right\} .
\end{aligned}
$$

Since $\sigma_{1}=\sigma_{2}=\sigma$ in $\Omega_{c}, \sigma_{2}=0$ in $\Omega_{1}$ and $\sigma_{1}=0$ in $\Omega_{2}$, it is easy to check that

$$
\begin{gathered}
\operatorname{Re}[c(\phi, \phi)]=\left\|\frac{\partial \varphi}{\partial x_{2}}\right\|_{L^{2}\left(\Omega_{1} \cup \Omega_{c}\right)}^{2}+\left\|\frac{\partial \varphi}{\partial x_{1}}\right\|_{L^{2}\left(\Omega_{2} \cup \Omega_{c}\right)}^{2}+\frac{1}{1+\sigma^{2}} \sum_{j=1}^{2}\left\|\frac{\partial \varphi}{\partial x_{j}}\right\|_{L^{2}\left(\Omega_{j}\right)}^{2} \\
+\sigma^{2}\|k \varphi\|_{L^{2}\left(\Omega_{c}\right)}^{2}-\|k \varphi\|_{L^{2}\left(\Omega_{\mathrm{PML}}\right)}^{2}-Z\|\varphi\|_{L^{2}\left(\Sigma_{\mathrm{PML}}\right)^{\prime}}^{2} \\
\frac{\operatorname{Im}[c(\varphi, \varphi)]}{\sigma}=\left\|\frac{\partial \varphi}{\partial x_{2}}\right\|_{L^{2}\left(\Omega_{1}\right)}^{2}+\left\|\frac{\partial \varphi}{\partial x_{1}}\right\|_{L^{2}\left(\Omega_{2}\right)}^{2}-\frac{1}{1+\sigma^{2}} \sum_{j=1}^{2}\left\|\frac{\partial \varphi}{\partial x_{j}}\right\|_{L^{2}\left(\Omega_{j}\right)}^{2} \\
-2\|k \varphi\|_{L^{2}\left(\Omega_{c}\right)}^{2}-\|k \varphi\|_{L^{2}\left(\Omega_{1} \cup \Omega_{2}\right)}^{2}-Z\|\varphi\|_{L^{2}\left(\Sigma_{\mathrm{PML}}\right)}^{2} .
\end{gathered}
$$

Then we observe that, for any $\gamma>0$,

$$
\begin{aligned}
& \operatorname{Re}[c(\varphi, \varphi)]+\frac{\gamma-1}{\sigma} \operatorname{Im}[c(\varphi, \varphi)] \\
& =\|\nabla \varphi\|_{L^{2}\left(\Omega_{c}\right)}^{2}+\gamma\left\|\frac{\partial \varphi}{\partial x_{2}}\right\|_{L^{2}\left(\Omega_{1}\right)}^{2}+\gamma\left\|\frac{\partial \varphi}{\partial x_{1}}\right\|_{L^{2}\left(\Omega_{2}\right)}^{2}+\frac{2-\gamma}{1+\sigma^{2}} \sum_{j=1}^{2}\left\|\frac{\partial \varphi}{\partial x_{j}}\right\|_{L^{2}\left(\Omega_{j}\right)}^{2} \\
& \quad+\left(1+\sigma^{2}-2 \gamma\right)\|k \varphi\|_{L^{2}\left(\Omega_{c}\right)}^{2}-\gamma\|k \varphi\|_{L^{2}\left(\Omega_{1} \cup \Omega_{2}\right)}^{2}-\gamma Z\|\varphi\|_{L^{2}\left(\Sigma_{\mathrm{PML}}\right)}^{2} .
\end{aligned}
$$


Since $\varphi=0$ on $\Gamma_{2} \cup \Gamma_{1}$, from the trace theorem we deduce easily that

$$
\|\varphi\|_{L^{2}\left(\Sigma_{\mathrm{PML}}\right)}^{2} \leq 2 d\left\|\frac{\partial \varphi}{\partial x_{2}}\right\|_{L^{2}\left(\Omega_{1} \cup \Omega_{c}\right)}^{2}, \quad\|\varphi\|_{L^{2}\left(\Omega_{j}\right)}^{2} \leq d^{2}\left\|\frac{\partial \varphi}{\partial x_{j}}\right\|_{L^{2}\left(\Omega_{j}\right)}^{2}, \quad j=1,2 .
$$

Substitute the above estimates into (5.10) we obtain

$$
\begin{aligned}
& \operatorname{Re}[c(\varphi, \varphi)]+\frac{\gamma-1}{\sigma} \operatorname{Im}[c(\varphi, \varphi)] \\
& \geq\|\nabla \varphi\|_{L^{2}\left(\Omega_{c}\right)}^{2}+\gamma\left\|\frac{\partial \varphi}{\partial x_{2}}\right\|_{L^{2}\left(\Omega_{1}\right)}^{2}+\gamma\left\|\frac{\partial \varphi}{\partial x_{1}}\right\|_{L^{2}\left(\Omega_{2}\right)}^{2} \\
& \quad+\left(\frac{2-\gamma}{1+\sigma^{2}}-2 \gamma k^{2} d^{2}-2 \gamma Z d\right) \sum_{j=1}^{2}\left\|\frac{\partial \varphi}{\partial x_{j}}\right\|_{L^{2}\left(\Omega_{j}\right)}^{2}-2 \gamma Z d\left\|\frac{\partial \varphi}{\partial x_{2}}\right\|_{L^{2}\left(\Omega_{c}\right)}^{2} \\
& \quad+\left(1+\sigma^{2}-2 \gamma\right)\|k \varphi\|_{L^{2}\left(\Omega_{c}\right)}^{2}+\gamma\|k \varphi\|_{L^{2}\left(\Omega_{1} \cup \Omega_{2}\right)}^{2} .
\end{aligned}
$$

Now taking $\gamma=\left[4+4\left(k^{2} d^{2}+Z d+1\right)\left(1+\sigma^{2}\right)\right]^{-1}$, we have

$$
] \frac{2-\gamma}{1+\sigma^{2}}-2 \gamma k^{2} d^{2}-2 \gamma Z d \geq \frac{1}{1+\sigma^{2}} \geq \gamma, \quad 1+\sigma^{2}-2 \gamma \geq \gamma, \quad \gamma Z d<\frac{1}{4} .
$$

Since $0<\gamma<1$ and $\sigma \geq 1$, we have

$$
2|c(\varphi, \varphi)| \geq \operatorname{Re}[c(\varphi, \varphi)]+(\gamma-1) \sigma^{-1} \operatorname{Im}[c(\varphi, \varphi)] \geq \gamma\|\| \varphi \|_{H^{1}\left(\Omega_{\mathrm{PML}}\right)}^{2} .
$$

This completes the proof.

\subsection{Convergence analysis for the PML method}

Now we present the main results of this paper.

Lemma 5.2. Let (H1)-(H3) be satisfied. Then for any $f \in H^{1 / 2}\left(\Gamma_{1}\right)$,

$$
\|(T-\hat{T}) f\|_{H^{-\frac{1}{2}\left(\Gamma_{1}\right)}} \leq C \hat{C}_{\mathrm{IS}}^{-1} \bar{\sigma}\left(\sigma^{2}+d Z\right)^{2}\left(\zeta_{p} e^{-\frac{1}{2} \gamma_{0} k \bar{\sigma}}+\zeta_{s} e^{-Z\left(L_{2}+d_{2}\right)}\right)\|f\|_{H^{\frac{1}{2}\left(\Gamma_{1}\right)}} .
$$

Proof. Let $w$ be the solution of (5.5) and define

$$
X(f):=\left\{\zeta \in H^{1}\left(\Omega_{\mathrm{PML}}\right): \zeta=0 \text { on } \Gamma_{1} \text { and } \zeta=\mathbb{E}(f) \text { on } \Gamma_{2}\right\} .
$$

We claim that there exists a constant $C>0$ independent of $\Omega_{\mathrm{PML}}$ such that

$$
\inf _{\zeta \in X(f)}\|\zeta\|_{H^{1}\left(\Omega_{\mathrm{PML}}\right)} \leq C\|\mathbb{E}(f)\|_{H^{1 / 2}\left(\Gamma_{2}\right)} .
$$

In fact, by the Sobolev extension theorem, we can extend $\mathbb{E}(f)$ by $\tilde{f}$ as follows

$$
\tilde{f} \in H^{1 / 2}\left(\widetilde{\Gamma}_{2}\right), \quad \tilde{f}=\mathbb{E}(f) \quad \text { on } \Gamma_{2}, \quad \text { and } \quad\|\tilde{f}\|_{H^{1 / 2}\left(\widetilde{\Gamma}_{2}\right)} \leq C\|\mathbb{E}(f)\|_{H^{1 / 2}\left(\Gamma_{2}\right)},
$$


where $\widetilde{\Gamma}_{2}:=\partial \widetilde{B}_{2}$ is the boundary of the box $\widetilde{B}_{2}:=\left\{x \in \mathbb{R}^{2}:\left|x_{1}\right|<L_{1}+d_{1},\left|x_{2}\right|<L_{2}+d_{2}\right\}$. The weighted $H^{\frac{1}{2}}$-norm in (4.34) indicates that the constant $C$ is independent of $\Gamma_{2}$. Then (5.12) follows from (5.13) and the following trace inequality:

$$
\inf _{\zeta \in X(f)}\|\zeta\|\left\|_{H^{1}\left(\Omega_{\mathrm{PML}}\right)} \leq \inf _{\substack{v \in H^{1}\left(\widetilde{B}_{2}\right) \\ v=\tilde{f} \text { on } \widetilde{\Gamma}_{2}, v=0 \text { in } \Omega_{1}}}\right\| v\left\|_{H^{1}\left(\widetilde{B}_{2}\right)} \leq C\right\| \tilde{f} \|_{H^{1 / 2}\left(\widetilde{\Gamma}_{2}\right)} .
$$

Since $\varphi=w-\zeta \in H_{\Gamma_{1} \cup \Gamma_{2}}^{1}\left(\Omega_{\mathrm{PML}}\right)$ for any $\zeta \in X(f)$, by (5.8) and (5.11), we have

$$
\begin{aligned}
& \hat{C}_{\mathrm{IS}}\|w-\zeta\|_{H^{1}\left(\Omega_{\mathrm{PML}}\right)}^{2} \leq 24|c(w-\zeta, \varphi)|=24|c(\zeta, \varphi)| \\
\leq & {\left[24(1+\sigma)^{2}+2 d Z\right]\left\|\left|\zeta\left\|_{H^{1}\left(\Omega_{\mathrm{PML}}\right)}|| \varphi \mid\right\|_{H^{1}\left(\Omega_{\mathrm{PML}}\right)} .\right.\right.}
\end{aligned}
$$

This proves

$$
\||w|\|_{H^{1}\left(\Omega_{\mathrm{PML}}\right)} \leq C \hat{C}_{\mathrm{IS}}^{-1}\left(\sigma^{2}+d Z\right) \inf _{\zeta \in X(f)}\|\zeta\|_{H^{1}\left(\Omega_{\mathrm{PML}}\right)} .
$$

Now we test (5.5a) with any $\varphi \in H_{\Gamma_{2}}^{1}\left(\Omega_{\mathrm{PML}}\right)$ and obtain

$$
\left|\left\langle\frac{\partial w}{\partial n}, \varphi\right\rangle_{\Gamma_{1}}\right| \leq|c(w, \varphi)| \leq C\left(\sigma^{2}+d Z\right)\||w|\|_{H^{1}\left(\Omega_{\mathrm{PML}}\right)}\|\mid \varphi\|_{H^{1}\left(\Omega_{\mathrm{PML}}\right)}
$$

where we have used (5.11) in the second inequality. Thus by (5.12), (5.14) we have

$$
\|(T-\hat{T}) f\|_{H^{-1 / 2}\left(\Gamma_{1}\right)}=\left\|\frac{\partial w}{\partial n}\right\|_{H^{-1 / 2}\left(\Gamma_{1}\right)} \leq C \hat{C}_{\mathrm{IS}}^{-1}\left(\sigma^{2}+d Z\right)^{2}\|\mathbb{E}(f)\|_{H^{1 / 2}\left(\Gamma_{2}\right)} .
$$

We complete the proof by using Theorem 4.1 and (H3).

Theorem 5.1. Let (H1)-(H3) be satisfied and let $u$ be the solution of (1.1). For sufficiently large $\bar{\sigma} \geq \bar{\sigma}_{0}$, where $\bar{\sigma}_{0}>0$ is a constant independent of $k$ and $Z$, the UPML problem (5.1) has a unique solution $\hat{u}$. Moreover,

$$
\|u-\hat{u}\|_{H^{1}\left(\Omega_{1}\right)} \leq C C_{\mathrm{IS}}^{-1} \hat{C}_{\mathrm{IS}}^{-1} \zeta\left(\zeta_{p} e^{-\frac{1}{2} \gamma_{0} k \bar{\sigma}}+\zeta_{s} e^{-Z\left(L_{2}+d_{2}\right)}\right)\|\hat{u}\|_{H^{1 / 2}\left(\Gamma_{1}\right)}
$$

where $\zeta:=\bar{\sigma}\left(\sigma^{2}+d Z\right)^{2}$ and $C_{\mathrm{IS}}^{-1}, \hat{C}_{\mathrm{IS}}^{-1}$ are respectively the inf-sup constants in (3.14) and (5.8).

Proof. We prove the estimate (5.16) first. Suppose that the solution $\hat{u}$ of (5.1) exists. By (3.13) and (5.2), simple integration by parts implies

$$
a(u-\hat{u}, \varphi)=\hat{a}(\hat{u}, \varphi)-a(\hat{u}, \varphi)=\langle T \hat{u}-\hat{T} \hat{u}, \varphi\rangle_{\Gamma_{1}}, \quad \forall \varphi \in H_{\Gamma_{D}}^{1}\left(\Omega_{1}\right) .
$$

Using (3.14) and Lemma 5.2, we obtain (5.16).

Now we turn to the well-posedness of the UPML problem. By the Fredholm alternative theorem we only need to show the uniqueness of $\hat{u}$. For that purpose we assume 
(5.1) has a solution $\hat{u}$ for $g=0$. By the uniqueness of the solution of (1.1), we know that the corresponding scattering solution $u=0$ in $\Omega_{1}$. Thus (5.16) implies

$$
\|\hat{u}\|_{H^{1}\left(\Omega_{1}\right)} \leq C C_{\mathrm{IS}}^{-1} \hat{C}_{\mathrm{IS}}^{-1} \zeta\left(\zeta_{p} e^{-\frac{1}{2} \gamma_{0} k \bar{\sigma}}+\zeta_{s} e^{-Z\left(L_{2}+d_{2}\right)}\right)\|\hat{u}\|_{H^{1 / 2}\left(\Gamma_{1}\right)} .
$$

Thus for sufficiently large $\bar{\sigma}$ we conclude that $\hat{u}=0$ on $\Omega_{1}$. That $\hat{u}$ also vanishes in $\Omega_{\text {PML }}$ follows from Lemma 5.1 since $\hat{u}$ satisfies the PML equation (5.4) with homogeneous boundary conditions.

\section{Appendix}

\section{A Some technical proofs}

In this section, we present the proofs for some technical estimates which are used in our analysis.

Lemma A.1. Let (H1)-(H2) be satisfied. Let $\tilde{a}, \tilde{b}, \tilde{\rho}, \tilde{b}_{\delta}$ and $\tilde{\rho}_{\delta}$ be defined in (4.5) and (4.8) for any $\boldsymbol{x} \in \Gamma_{2}, \boldsymbol{y} \in \bar{\Omega}_{1}$. Assume $b_{1} \leq \delta a_{1}$. Then

$$
\frac{\delta \bar{\sigma}}{\sqrt{2+\delta^{2}}} \leq b_{2}^{\delta} \leq 2^{1 / 4} \delta \bar{\sigma} \quad \text { and } \quad 2^{-1 / 4} \delta a_{1} \leq b_{1}^{\delta} \leq \delta a_{1} \sqrt{2+\delta^{2}} .
$$

Proof. Let $\tilde{\boldsymbol{x}}_{\delta}=\left(\tilde{x}_{1}, \sqrt{1+1 / \delta^{2}} \tilde{x}_{2}\right), \tilde{y}_{\delta}=\left(\tilde{y}_{1},-\sqrt{1+1 / \delta^{2}} \tilde{y}_{2}\right)$, then

$$
\begin{aligned}
\tilde{b}_{\delta} & =\left(\tilde{b}^{2}+\delta^{2} \tilde{\rho}^{2}\right)^{1 / 2}=\left(\left(\tilde{x}_{2}+\tilde{y}_{2}\right)^{2}+\delta^{2}\left(\tilde{x}_{1}-\tilde{y}_{1}\right)^{2}+\delta^{2}\left(\tilde{x}_{2}+\tilde{y}_{2}\right)^{2}\right)^{1 / 2} \\
& =\delta\left(\left(\tilde{x}_{1}-\tilde{y}_{1}\right)^{2}+\left(1+1 / \delta^{2}\right)\left(\tilde{x}_{2}+\tilde{y}_{2}\right)^{2}\right)^{1 / 2}=\delta \rho\left(\tilde{x}_{\delta}, \tilde{y}_{\delta}\right) .
\end{aligned}
$$

Since $b_{1} \leq \delta a_{1}$, namely, $\left|x_{2}+y_{2}\right| \leq \delta\left|x_{1}-y_{1}\right|$, it is easy to see that $b_{2}=0, a_{2}=\bar{\sigma}$ and $a_{1} \geq d_{1}>0$. By (H2), it is easy to check that $\bar{\sigma} \geq 2 a_{1}$. Then

$$
\begin{aligned}
\left|\rho\left(\tilde{\boldsymbol{x}}_{\delta}, \tilde{\boldsymbol{y}}_{\delta}\right)\right|^{2} & =\left|\left(\tilde{x}_{1}-\tilde{y}_{1}\right)^{2}+\left(1+1 / \delta^{2}\right)\left(\tilde{x}_{2}+\tilde{y}_{2}\right)^{2}\right| \\
& =\sqrt{\left(a_{1}^{2}-\bar{\sigma}^{2}+\left(1+1 / \delta^{2}\right) b_{1}^{2}\right)^{2}+4\left(a_{1} \bar{\sigma}\right)^{2}} \\
& \leq \sqrt{\left(\bar{\sigma}^{2}-\left(2+\delta^{2}\right) a_{1}^{2}\right)^{2}+4\left(a_{1} \bar{\sigma}\right)^{2}} \leq \sqrt{2} \bar{\sigma}^{2} .
\end{aligned}
$$

By Lemma 4.1 and $b_{1} \leq \delta a_{1}$, we have

$$
\operatorname{Im} \rho\left(\tilde{\boldsymbol{x}}_{\delta}, \tilde{\boldsymbol{y}}_{\delta}\right) \geq \frac{\left|x_{1}-y_{1}\right| \bar{\sigma}}{\sqrt{\left|x_{1}-y_{1}\right|^{2}+\left(1+1 / \delta^{2}\right)\left|x_{2}+y_{2}\right|^{2}}} \geq \frac{\bar{\sigma}}{\sqrt{2+\delta^{2}}} .
$$

From the last two inequalities, we find that

$$
\frac{\bar{\sigma}}{\sqrt{2+\delta^{2}}} \leq \operatorname{Im} \rho\left(\tilde{\boldsymbol{x}}_{\delta}, \tilde{\boldsymbol{y}}_{\delta}\right) \leq 2^{1 / 4} \bar{\sigma}
$$


Note that $\operatorname{Re} \rho\left(\tilde{\boldsymbol{x}}_{\delta}, \tilde{\boldsymbol{y}}_{\delta}\right) \cdot \operatorname{Im} \rho\left(\tilde{\boldsymbol{x}}_{\delta}, \tilde{\boldsymbol{y}}_{\delta}\right)=a_{1} \bar{\sigma}$, we deduce that

$$
2^{-1 / 4} a_{1} \leq \operatorname{Re} \rho\left(\tilde{\boldsymbol{x}}_{\delta}, \tilde{\boldsymbol{y}}_{\delta}\right) \leq a_{1} \sqrt{2+\delta^{2}} .
$$

Then the proof is completed by combining (A.2)-(A.3) with (A.1).

Lemma A.2. Let (H1)-(H2) be satisfied. For any $x \in \Gamma_{2}, y \in \bar{\Omega}_{1}$, define

$$
\xi=\frac{k}{\tilde{\rho}_{\delta}}\left(\tilde{a} t-\mathbf{i} \tilde{b}_{\delta} \sqrt{t^{2}-1}\right), \quad \forall t \in[1, \infty) .
$$

If $b_{1} \leq \delta a_{1}$, then $\operatorname{Im} \xi<0$ for all $t \geq \sqrt{2}$.

Proof. From the definition, it is easy to see that

$$
\operatorname{Im} \xi=\frac{k}{\left|\tilde{\rho}_{\delta}\right|^{2}}\left[t\left(\rho_{1}^{\delta} a_{2}-\rho_{2}^{\delta} a_{1}\right)-t^{\prime}\left(\rho_{1}^{\delta} b_{1}^{\delta}+\rho_{2}^{\delta} b_{2}^{\delta}\right)\right] .
$$

For $\rho_{1}^{\delta} a_{2} \leq \rho_{2}^{\delta} a_{1}$, using Lemma A.1 and (4.10), we have for $t \geq \sqrt{2}$,

$$
\operatorname{Im} \xi \leq-\frac{k}{\left|\tilde{\rho}_{\delta}\right|^{2}} t^{\prime}\left(\rho_{1}^{\delta} b_{1}^{\delta}+\rho_{2}^{\delta} b_{2}^{\delta}\right) \leq-\frac{k}{\left|\tilde{\rho}_{\delta}\right|^{2}} \frac{\delta \bar{\sigma}}{\sqrt{2+\delta^{2}}} \sqrt{1+\delta^{2}} \gamma_{0} \bar{\sigma}<0 .
$$

For $\rho_{1}^{\delta} a_{2}>\rho_{2}^{\delta} a_{1}$, we write

$$
\frac{\left|\tilde{\rho}_{\delta}\right|^{2}}{k t} \operatorname{Im} \xi=M_{1}-M_{2}
$$

with $M_{1}=\rho_{1}^{\delta} a_{2}$ and

$$
M_{2}=\rho_{2}^{\delta} a_{1}+\frac{t^{\prime}}{t}\left(\rho_{1}^{\delta} b_{1}^{\delta}+\rho_{2}^{\delta} b_{2}^{\delta}\right) .
$$

Obviously, $M_{1}>0$ and $M_{2}>0$.

Noting that $\left(t^{\prime}\right)^{2} / t^{2} \geq 1 / 2$ for $t \geq \sqrt{2}$, we have

$$
\begin{aligned}
2\left(M_{1}^{2}-M_{2}^{2}\right) & \leq 2\left[\left(\rho_{1}^{\delta}\right)^{2} a_{2}^{2}-\left(\rho_{2}^{\delta}\right)^{2} a_{1}^{2}-\frac{1}{2}\left(\left(\rho_{1}^{\delta}\right)^{2}\left(b_{1}^{\delta}\right)^{2}+\left(\rho_{2}^{\delta}\right)^{2}\left(b_{2}^{\delta}\right)^{2}\right)\right] \\
& =|\tilde{a}|^{2} \operatorname{Re} \tilde{\rho}_{\delta}^{2}-\left|\tilde{\rho}_{\delta}\right|^{2} \operatorname{Re} \tilde{a}^{2}-\frac{1}{2}\left(\left|\tilde{\rho}_{\delta}\right|^{2}\left|\tilde{b}_{\delta}\right|^{2}+\operatorname{Re} \tilde{\rho}_{\delta}^{2} \operatorname{Re} \tilde{b}_{\delta}^{2}\right) \\
& \leq\left|\tilde{\rho}_{\delta}\right|^{2} \operatorname{Re} \tilde{b}_{\delta}^{2}-\left|\tilde{b}_{\delta}\right|^{2} \operatorname{Re} \tilde{\rho}_{\delta}^{2}-\frac{1}{2}\left(\left|\tilde{\rho}_{\delta}\right|^{2}\left|\tilde{b}_{\delta}\right|^{2}+\operatorname{Re} \tilde{\rho}_{\delta}^{2} \operatorname{Re} \tilde{b}_{\delta}^{2}\right) \\
& \leq \frac{1}{2}\left|\tilde{\rho}_{\delta}\right|^{2}\left|\tilde{b}_{\delta}\right|^{2}-\frac{1}{2} \operatorname{Re} \tilde{b}_{\delta}^{2}\left(\operatorname{Re} \tilde{\rho}_{\delta}^{2}-2\left|\tilde{\rho}_{\delta}\right|^{2}\right) \\
& \leq \frac{1}{2}\left|\tilde{\rho}_{\delta}\right|^{2}\left(\left|\tilde{b}_{\delta}\right|^{2}+2 \operatorname{Re} \tilde{b}_{\delta}^{2}\right) .
\end{aligned}
$$


Using Lemma A.1 and (H2),

$$
\left|\tilde{b}_{\delta}\right|^{2}+2 \operatorname{Re} \tilde{b}_{\delta}^{2}=\left(b_{1}^{\delta}\right)^{2}+\left(b_{2}^{\delta}\right)^{2}+2\left[\left(b_{1}^{\delta}\right)^{2}-\left(b_{2}^{\delta}\right)^{2}\right]=3\left(b_{1}^{\delta}\right)^{2}-\left(b_{2}^{\delta}\right)^{2}<0 .
$$

Thus

$$
2\left(M_{1}^{2}-M_{2}^{2}\right) \leq \frac{1}{2}\left|\tilde{\rho}_{\delta}\right|^{2}\left(\left|\tilde{b}_{\delta}\right|^{2}+2 \operatorname{Re} \tilde{b}_{\delta}^{2}\right)<0
$$

Therefore,

$$
\frac{\left|\tilde{\rho}_{\delta}\right|^{2}}{k t}\left(M_{1}+M_{2}\right) \operatorname{Im} \xi=M_{1}^{2}-M_{2}^{2}<0
$$

The proof is completed.

The purpose of the following lemma is to prove inequality (4.19) in Lemma 4.4.

Lemma A.3. Let the assumptions in Lemma 4.4 be satisfied and let $f(t)$ be defined by (4.18), namely,

$$
f(t)=-\mathbf{i}\left(\frac{\mu_{+}(t)}{Z+\mathbf{i} \mu_{+}(t)}+\frac{\mu_{-}(t)}{Z+\mathbf{i} \mu_{-}(t)}\right) .
$$

Then

$$
|f(t)| \leq 1+C \bar{\sigma}(d+\bar{\sigma}) .
$$

Proof. For convenience we denote $t^{\prime}=\sqrt{t^{2}-1}$ for any $t \geq 1$. From (4.12) and (4.6), we have

$$
\begin{aligned}
|\tilde{\rho}| \cdot\left|Z+\mathbf{i} \mu_{+}\right| & =\left|Z \tilde{\rho}+\mathbf{i} k \tilde{b} t+k \tilde{a} t^{\prime}\right| \\
& =\left|Z \rho_{1}+\mathbf{i} Z \rho_{2}+\mathbf{i} k b_{1} t-k b_{2} t+k a_{1} t^{\prime}+\mathbf{i} k a_{2} t^{\prime}\right| \\
& \geq Z \rho_{2}+k b_{1} t+k a_{2} t^{\prime} \geq Z \gamma_{0} \bar{\sigma} .
\end{aligned}
$$

Next we estimate $|\tilde{\rho}| \cdot\left|Z+\mathbf{i} \mu_{-}\right|$. Note that

$$
\begin{aligned}
& |\tilde{\rho}| \cdot\left|Z+\mathbf{i} \mu_{-}\right|=\left|Z \tilde{\rho}+\mathbf{i} k \tilde{b} t-k \tilde{a} t^{\prime}\right| \\
= & \left|Z \rho_{1}-k b_{2} t-k a_{1} t^{\prime}+\mathbf{i}\left(Z \rho_{2}+k b_{1} t-k a_{2} t^{\prime}\right)\right| .
\end{aligned}
$$

Let $\Gamma_{2, \|}$ be the part of $\Gamma_{2}$ parallel to the horizontal direction and $\Gamma_{2, \perp}$ be the part of $\Gamma_{2}$ perpendicular to the horizontal direction, respectively.

For any $\boldsymbol{x} \in \Gamma_{2, \|}$, we have $b_{2}=\bar{\sigma}$. By (4.7) and (H2), we have

$$
\begin{aligned}
|\tilde{\rho}| \cdot\left|Z+\mathbf{i} \mu_{-}\right| & \geq\left|k b_{2} t+k a_{1} t^{\prime}-Z \rho_{1}\right| \\
& \geq k \bar{\sigma}-Z \sqrt{\left(2 L_{1}+d_{1}\right)^{2}+\left(2 L_{2}+d_{2}\right)^{2}} \\
& \geq k \bar{\sigma} / 3 .
\end{aligned}
$$


For any $x \in \Gamma_{2, \perp}$, we have $a_{2}=\bar{\sigma}$. Let $Z \rho_{2}+k b_{1} t-k a_{2} t^{\prime}=0$, then $t^{\prime}=\frac{Z \rho_{2}+k b_{1} t}{k a_{2}}$. Insert it into (A.6) to get

$$
|\tilde{\rho}| \cdot\left|Z+\mathbf{i} \mu_{-}\right|=\left|Z \rho_{1}-k b_{2} t-k a_{1} t^{\prime}\right|=\left|k t\left(b_{2}+\frac{a_{1}}{a_{2}} b_{1}\right)-Z\left(\rho_{1}-\frac{a_{1}}{a_{2}} \rho_{2}\right)\right| .
$$

In the following, we prove that $\left|Z\left(\rho_{1}-\frac{a_{1}}{a_{2}} \rho_{2}\right)\right|$ is much smaller than $k t\left(b_{2}+\frac{a_{1}}{a_{2}} b_{1}\right)$, so there exists a positive lower bound of $|\tilde{\rho}| \cdot\left|Z+\mathbf{i} \mu_{-}\right|$. We shall consider two cases.

Case 1. Suppose $\frac{Z}{k} \sqrt{a_{1}^{2}+b_{1}^{2}} \leq b_{2} \leq \bar{\sigma}$. Then

$$
k t\left(b_{2}+\frac{a_{1}}{a_{2}} b_{1}\right) \geq k b_{2}+k \frac{a_{1}}{a_{2}} b_{1} \geq Z \sqrt{a_{1}^{2}+b_{1}^{2}}+k \frac{a_{1}}{a_{2}} b_{1} .
$$

From (4.6) and (4.7), it is easy to see that

$$
Z\left(\rho_{1}-\frac{a_{1}}{a_{2}} \rho_{2}\right) \leq Z \sqrt{a_{1}^{2}+b_{1}^{2}}-Z d_{1} \gamma_{0}
$$

where we have used that $a_{1}=\left|x_{1}-y_{1}\right| \geq d_{1}$ and $a_{2}=\bar{\sigma}$ on $\Gamma_{2, \perp}$.

Inserting (A.9) and (A.10) into (A.8), we obtain

$$
|\tilde{\rho}| \cdot\left|Z+\mathbf{i} \mu_{-}\right| \geq k \frac{a_{1}}{a_{2}} b_{1}+Z d_{1} \gamma_{0} \geq Z d_{1} \gamma_{0} .
$$

Case 2. Suppose $0 \leq b_{2}<\frac{Z}{k} \sqrt{a_{1}^{2}+b_{1}^{2}}$. Note that

$$
Z\left(\rho_{1}-\frac{a_{1}}{a_{2}} \rho_{2}\right)=\frac{Z\left(a_{2} \rho_{1}-a_{1} \rho_{2}\right)}{a_{2}}=\frac{Z\left(a_{2}^{2} \rho_{1}^{2}-a_{1}^{2} \rho_{2}^{2}\right)}{a_{2}\left(a_{1} \rho_{2}+a_{2} \rho_{1}\right)} .
$$

Direct computations yield that

$$
\begin{aligned}
a_{2}^{2} \rho_{1}^{2}-a_{1}^{2} \rho_{2}^{2} & =a_{2}^{2}\left(\left|\tilde{\rho}^{2}\right|+\operatorname{Re} \tilde{\rho}^{2}\right) / 2-a_{1}^{2}\left(\left|\tilde{\rho}^{2}\right|-\operatorname{Re} \tilde{\rho}^{2}\right) / 2 \\
& =\frac{1}{2}\left[\left|\tilde{\rho}^{2}\right|\left(a_{2}^{2}-a_{1}^{2}\right)+\operatorname{Re} \tilde{\rho}^{2}\left(a_{1}^{2}+a_{2}^{2}\right)\right] \\
& =\frac{1}{2} \frac{\left(a_{2}^{2}-a_{1}^{2}\right)^{2}\left(\left|\operatorname{Re} \tilde{\rho}^{2}\right|^{2}+\left|\operatorname{Im} \tilde{\rho}^{2}\right|^{2}\right)-\left(a_{1}^{2}+a_{2}^{2}\right)^{2}\left|\operatorname{Re} \tilde{\rho}^{2}\right|^{2}}{\left|\tilde{\rho}^{2}\right|\left(a_{2}^{2}-a_{1}^{2}\right)+\left|\operatorname{Re} \tilde{\rho}^{2}\right|\left(a_{1}^{2}+a_{2}^{2}\right)}=\frac{D}{H^{\prime}}
\end{aligned}
$$

where $D:=2\left(a_{2}^{2} b_{2}^{2}-a_{1}^{2} b_{1}^{2}\right)\left(a_{2}^{2} b_{1}^{2}-a_{1}^{2} b_{2}^{2}\right)+4 a_{1} a_{2}\left(a_{2}^{2}-a_{1}^{2}\right)\left(a_{2} b_{1}-a_{1} b_{2}\right)\left(a_{1} b_{1}+a_{2} b_{2}\right)$ and $H:=$ $\left|\tilde{\rho}^{2}\right|\left(a_{2}^{2}-a_{1}^{2}\right)+\left|\operatorname{Re} \tilde{\rho}^{2}\right|\left(a_{1}^{2}+a_{2}^{2}\right)$.

Using $a_{2}=\bar{\sigma}, b_{1}>\delta a_{1},(4.6)$ and (H2), we deduce that

$$
\begin{aligned}
& H=\left|\tilde{\rho}^{2}\right|\left(a_{2}^{2}-a_{1}^{2}\right)+\left|\operatorname{Re} \tilde{\rho}^{2}\right|\left(a_{1}^{2}+a_{2}^{2}\right) \geq \gamma_{0}^{2} \bar{\sigma}^{4} / 2, \\
& |D| \leq 2 a_{2}^{4} b_{1}^{2} b_{2}^{2}+2 a_{1}^{2} a_{2}^{2} b_{1}^{4}+4 a_{1} a_{2}^{4} b_{1}\left(a_{1} b_{1}+a_{2} b_{2}\right) .
\end{aligned}
$$


Using the above estimates, we obtain

$$
\left|a_{2}^{2} \rho_{1}^{2}-a_{1}^{2} \rho_{2}^{2}\right|=\left|\frac{D}{H}\right| \leq \frac{4 b_{1}^{2} b_{2}^{2}+4 b_{1}^{4}+8 a_{1}^{2} b_{1}^{2}+8 a_{1} a_{2} b_{1} b_{2}}{\gamma_{0}^{2}} .
$$

Substituting (A.13) into (A.12) yields

$$
\left|Z\left(\rho_{1}-\frac{a_{1}}{a_{2}} \rho_{2}\right)\right| \leq \frac{Z\left(4 b_{1}^{2} b_{2}^{2}+4 b_{1}^{4}+8 a_{1}^{2} b_{1}^{2}+8 a_{1} a_{2} b_{1} b_{2}\right)}{a_{1} \gamma_{0}^{3} \bar{\sigma}^{2}} .
$$

Then from (H2), $b_{1}>\delta a_{1}$ and $a_{1} \geq d_{1}$, we find that for any $x \in \Gamma_{2, \perp}$,

$$
\begin{aligned}
|\tilde{\rho}| \cdot\left|Z+\mathbf{i} \mu_{-}\right| & \geq k t\left(b_{2}+\frac{a_{1}}{a_{2}} b_{1}\right)-\left|Z\left(\rho_{1}-\frac{a_{1}}{a_{2}} \rho_{2}\right)\right| \\
& \geq \frac{Z}{a_{1} \gamma_{0}^{3} \bar{\sigma}^{2}}\left(\frac{k}{Z} \gamma_{0}^{3} \bar{\sigma} a_{1}\left(a_{1} b_{1}+a_{2} b_{2}\right)-\left(4 b_{1}^{2} b_{2}^{2}+4 b_{1}^{4}+8 a_{1}^{2} b_{1}^{2}+8 a_{1} a_{2} b_{1} b_{2}\right)\right) \\
& \geq \frac{k a_{1} b_{1}}{9 \bar{\sigma}}+\frac{k b_{2}}{9} \geq \frac{k \delta d_{1}^{2}}{9 \bar{\sigma}} .
\end{aligned}
$$

Finally, from (A.5), (A.7), (A.11) and (A.14), we conclude that

$$
\left|\frac{\mu_{ \pm}(t)}{Z+\mathbf{i} \mu_{ \pm}(t)}\right| \leq 1+\left|\frac{Z}{Z+\mathbf{i} \mu_{ \pm}(t)}\right| \leq 1+C \bar{\sigma}|\tilde{\rho}| \leq 1+C \bar{\sigma}(d+\bar{\sigma}) .
$$

Then the proof is completed.

Lemma A.4. Let the assumptions in Lemma 4.5 be satisfied and let $\xi_{ \pm}(t)$ be defined by (4.11). Then

$$
\left|\frac{1}{Z+\mathbf{i} \mu\left(\xi_{ \pm}(t)\right)}\right| \leq C(d+\bar{\sigma})
$$

Proof. From (4.10) and (4.12), we find that

$$
\begin{aligned}
\left|\tilde{\rho}_{\delta}\right| \cdot\left|Z+\mathbf{i} \mu_{+}\right| & =\left|Z \tilde{\rho}_{\delta}+\mathbf{i} k \tilde{b}_{\delta} t+k \tilde{a} t^{\prime}\right| \\
& =\left|Z \rho_{1}^{\delta}+\mathbf{i} Z \rho_{2}^{\delta}+\mathbf{i} k b_{1}^{\delta} t-k b_{2}^{\delta} t+k a_{1} t^{\prime}+\mathbf{i} k a_{2} t^{\prime}\right| \\
& \geq Z \rho_{2}^{\delta}+k b_{1}^{\delta} t+k a_{2} t^{\prime} \geq Z \sqrt{1+\delta^{2}} \gamma_{0} \bar{\sigma} .
\end{aligned}
$$

Now we estimate $\left|\tilde{\rho}_{\delta}\right| \cdot\left|Z+\mathbf{i} \mu_{-}\right|$. Note that

$$
\begin{aligned}
\left|\tilde{\rho}_{\delta}\right| \cdot\left|Z+\mathbf{i} \mu_{-}\right| & =\left|Z \tilde{\rho}_{\delta}+\mathbf{i} k \tilde{b}_{\delta} t-k \tilde{a} t^{\prime}\right| \\
& =\left|Z \rho_{1}^{\delta}-k b_{2}^{\delta} t-k a_{1} t^{\prime}+\mathbf{i}\left(Z \rho_{2}^{\delta}+k b_{1}^{\delta} t-k a_{2} t^{\prime}\right)\right| .
\end{aligned}
$$

Since $b_{1} \leq \delta a_{1}$, by (H2), it is easy to see that $x \in \Gamma_{2, \perp}$ and

$$
b_{2}=0, \quad a_{2}=\bar{\sigma}, \quad a_{1} \geq d_{1} .
$$


Using (H2), (A.17) and Lemma A.1, by arguments similar to Case 2 in Lemma A.3 and careful calculations, we find that

$$
\begin{aligned}
\left|Z\left(\rho_{1}^{\delta}-\frac{a_{1}}{a_{2}} \rho_{2}^{\delta}\right)\right| \leq & \frac{4 Z}{a_{1} \gamma_{0}^{3} \bar{\sigma}^{6}}\left(a_{2}^{4}\left(b_{1}^{\delta}\right)^{2}\left(b_{2}^{\delta}\right)^{2}+a_{1}^{2} a_{2}^{2}\left(b_{2}^{\delta}\right)^{4}+2 a_{1}^{2} a_{2}^{4}\left(b_{1}^{\delta}\right)^{2}\right. \\
& \left.+2 a_{1} a_{2}^{5} b_{1}^{\delta} b_{2}^{\delta}+2 a_{1}^{3} a_{2}^{3} b_{1}^{\delta} b_{2}^{\delta}+2 a_{1}^{2} a_{2}^{4}\left(b_{2}^{\delta}\right)^{2}\right) \leq \frac{32 Z a_{1} \delta^{2}}{\gamma_{0}^{3}} .
\end{aligned}
$$

From (H2), it is easy to see that $\frac{k}{Z} \gamma_{0}^{3} \bar{\sigma} \geq 9 a_{1}$. Then using Lemma A.1 and (A.18), by careful calculations and arrangements, we deduce that

$$
\begin{aligned}
\left|\tilde{\rho}_{\delta}\right| \cdot\left|Z+\mathbf{i} \mu_{-}\right| & \geq k t\left(b_{2}^{\delta}+\frac{a_{1}}{a_{2}} b_{1}^{\delta}\right)-\left|Z\left(\rho_{1}^{\delta}-\frac{a_{1}}{a_{2}} \rho_{2}^{\delta}\right)\right| \\
& \geq \frac{Z \delta}{\gamma_{0}^{3} \sqrt{2+\delta^{2}}}\left(\frac{k}{Z} \gamma_{0}^{3} \bar{\sigma}-32 a_{1} \delta \sqrt{2+\delta^{2}}\right) \geq \frac{Z \delta d_{1}}{\gamma_{0}^{3}} .
\end{aligned}
$$

From (A.15) and (A.19), it yields that

$$
\left|\frac{1}{Z+\mathbf{i} \mu_{ \pm}(t)}\right| \leq C\left|\tilde{\rho}_{\delta}\right| \leq C(d+\bar{\sigma}) .
$$

Then the proof is completed.

Lemma A.5. Let the assumptions in Lemma 4.5 be satisfied. Then

$$
\left|\frac{1}{\mu(\xi(t))}\right| \leq C, \quad\left|\frac{1}{Z+\mathbf{i} \mu(\xi(t))}\right| \leq C,
$$

where $\xi(t)=k t+\xi_{0}$ and $\xi_{0}=\xi_{-}(\sqrt{2})=\frac{k}{\tilde{\rho}_{\delta}}\left(\sqrt{2} \tilde{a}-\mathbf{i} \tilde{b}_{\delta}\right)$.

Proof. From the definition, it is easy to see that

$$
\begin{aligned}
\xi_{0} & =\frac{k}{\tilde{\rho}_{\delta}}\left(\sqrt{2} \tilde{a}-\mathbf{i} \tilde{b}_{\delta}\right)=\frac{k}{\left|\tilde{\rho}_{\delta}\right|^{2}}\left(\rho_{1}^{\delta}-\mathbf{i} \rho_{2}^{\delta}\right)\left[\sqrt{2}\left(a_{1}+\mathbf{i} a_{2}\right)-\mathbf{i}\left(b_{1}^{\delta}+\mathbf{i} b_{2}^{\delta}\right)\right] \\
& =\frac{k}{\left|\tilde{\rho}_{\delta}\right|^{2}}\left[\rho_{1}^{\delta}\left(\sqrt{2} a_{1}+b_{2}^{\delta}\right)+\rho_{2}^{\delta}\left(\sqrt{2} a_{2}-b_{1}^{\delta}\right)+\mathbf{i}\left(\rho_{1}^{\delta}\left(\sqrt{2} a_{2}-b_{1}^{\delta}\right)-\rho_{2}^{\delta}\left(\sqrt{2} a_{1}+b_{2}^{\delta}\right)\right)\right] .
\end{aligned}
$$

From the assumptions in Lemma $4.5, a_{2}=\bar{\sigma}$ and $b_{2}=0$. Then using $(\mathrm{H} 2)$,

$$
\begin{aligned}
\left|\tilde{\rho}_{\delta}\right|^{2} & =\left(1+\delta^{2}\right)|\tilde{\rho}|^{2}=\left(1+\delta^{2}\right) \sqrt{\left(a_{1}^{2}-a_{2}^{2}+b_{1}^{2}-b_{2}^{2}\right)^{2}+4\left(a_{1} a_{2}+b_{1} b_{2}\right)^{2}} \\
& =\left(1+\delta^{2}\right) \sqrt{\left(a_{2}^{2}-a_{1}^{2}-b_{1}^{2}\right)^{2}+4 a_{1}^{2} a_{2}^{2}} \leq \sqrt{2}\left(1+\delta^{2}\right) \bar{\sigma}^{2} .
\end{aligned}
$$


Thus by (4.9), (4.10) and Lemma A.1, we find that

$$
\begin{aligned}
\operatorname{Re} \xi \geq \operatorname{Re} \xi_{0} & =\frac{k}{\left|\tilde{\rho}_{\delta}\right|^{2}}\left[\rho_{1}^{\delta}\left(\sqrt{2} a_{1}+b_{2}^{\delta}\right)+\rho_{2}^{\delta}\left(\sqrt{2} a_{2}-b_{1}^{\delta}\right)\right] \\
& >\frac{k}{\left(1+\delta^{2}\right) \sqrt{2} \bar{\sigma}^{2}}\left[\sqrt{1+\delta^{2}} \gamma_{0} \bar{\sigma}\left(\sqrt{2} \bar{\sigma}-\delta \sqrt{2+\delta^{2}} \bar{\sigma} / 2\right)\right] \\
& >\frac{k \gamma_{0}}{2 \sqrt{1+\delta^{2}}},
\end{aligned}
$$

where we have used that $\delta<\gamma_{0}^{2} / 20<1 / 2$.

By arguments similar to (A.18), we get

$$
\left|\rho_{2}^{\delta} a_{1}-\rho_{1}^{\delta} a_{2}\right|=\left|\frac{\left(\rho_{2}^{\delta}\right)^{2} a_{1}^{2}-\left(\rho_{1}^{\delta}\right)^{2} a_{2}^{2}}{\rho_{2}^{\delta} a_{1}+\rho_{1}^{\delta} a_{2}}\right| \leq \frac{32 a_{1} \delta^{2}}{\gamma_{0}^{3} \bar{\sigma}} .
$$

Then by (H2), (A.20) and Lemma A.1,

$$
\begin{aligned}
|\operatorname{Im} \xi|=\left|\operatorname{Im} \xi_{0}\right| & =\frac{1}{\left|\tilde{\rho}_{\delta}\right|^{2}}\left|\rho_{2}^{\delta}\left(\sqrt{2} a_{1}+b_{2}^{\delta}\right)-\rho_{1}^{\delta}\left(\sqrt{2} a_{2}-b_{1}^{\delta}\right)\right| \\
& \geq \frac{1}{\left(1+\delta^{2}\right) 2 \bar{\sigma}^{2}}\left(\gamma_{0} \bar{\sigma} \frac{\delta \bar{\sigma}}{\sqrt{2+\delta^{2}}}-\frac{32 a_{1} \delta^{2}}{\gamma_{0}^{3} \bar{\sigma}}\right) \\
& \geq \frac{\gamma_{0} \delta}{6\left(1+\delta^{2}\right)} .
\end{aligned}
$$

Using (A.21) and (A.22), it is easy to see that

$$
\left|\operatorname{Im} \xi^{2}\right|=|2 \operatorname{Re} \xi \cdot \operatorname{Im} \xi| \geq \frac{k \gamma_{0}^{2} \delta}{6\left(\sqrt{1+\delta^{2}}\right)^{3}} .
$$

By (A.23), we derive that

$$
\left|\mu^{2}\right|=\left|k^{2}-\xi^{2}\right| \geq\left|\operatorname{Im} \xi^{2}\right| \geq \frac{k \gamma_{0}^{2} \delta}{6\left(\sqrt{1+\delta^{2}}\right)^{3}} .
$$

Then there exists a constant $C>0$ only depending on $k$ such that

$$
\left|\frac{1}{\mu(\xi(t))}\right| \leq C
$$

Now we estimate $\left|\frac{1}{Z+\mathbf{i} \mu}\right|$. Using (A.23), we have

$$
\left|\frac{1}{Z+\mathbf{i} \mu}\right|=\left|\frac{Z-\mathbf{i} \mu}{Z^{2}+\mu^{2}}\right|=\left|\frac{Z-\mathbf{i} \mu}{Z^{2}+k^{2}-\xi^{2}}\right| \leq\left|\frac{Z-\mathbf{i} \mu}{\operatorname{Im} \xi^{2}}\right| \leq C Z+\left|\frac{\mu}{\operatorname{Im} \xi^{2}}\right| .
$$


Noting that

$$
\left|\frac{\mu}{\operatorname{Im} \xi^{2}}\right|^{2}=\frac{\left|k^{2}-\xi^{2}\right|}{\left|\operatorname{Im} \xi^{2}\right|^{2}} \leq C+\frac{\left|\xi^{2}\right|}{\left|\operatorname{Im} \xi^{2}\right|^{2}} \leq C+\frac{\sqrt{\left(\operatorname{Re} \tilde{\zeta}^{2}\right)^{2}+\left(\operatorname{Im} \xi^{2}\right)^{2}}}{\left|\operatorname{Im} \xi^{2}\right|^{2}} \leq C+\frac{\left|\operatorname{Re} \xi^{2}\right|}{\left|\operatorname{Im} \xi^{2}\right|^{2}} .
$$

Inserting $\operatorname{Re} \tilde{\xi}^{2}=k^{2} t^{2}+2 k t \operatorname{Re} \xi_{0}+\operatorname{Re} \tilde{\xi}_{0}^{2}$ and $\operatorname{Im} \tilde{\xi}^{2}=2 k t \operatorname{Im} \xi_{0}+\operatorname{Im} \tilde{\zeta}_{0}^{2}$ into the last inequality, we have for some constant $T_{0}>0$, if $t \leq T_{0}$,

$$
\left|\frac{\mu}{\operatorname{Im} \xi^{2}}\right|^{2} \leq C+\frac{\left|\operatorname{Re} \xi^{2}\right|}{\left|\operatorname{Im} \xi^{2}\right|^{2}} \leq C+\frac{k^{2} T_{0}^{2}+2 k T_{0}\left|\operatorname{Re} \xi_{0}\right|+\left|\operatorname{Re} \xi_{0}^{2}\right|}{\left(\operatorname{Im} \xi_{0}^{2}\right)^{2}} \leq C,
$$

and if $t>T_{0}$,

$$
\left|\frac{\mu}{\operatorname{Im} \xi^{2}}\right|^{2} \leq C+\frac{\left|\operatorname{Re} \xi^{2}\right|}{\left|\operatorname{Im} \xi^{2}\right|^{2}} \leq C+\frac{k^{2} t^{2}+2 k t\left|\operatorname{Re} \xi_{0}\right|+\left|\operatorname{Re} \xi_{0}^{2}\right|}{4 k^{2} t^{2}\left(\operatorname{Im} \xi_{0}\right)^{2}} \leq C .
$$

Here we have used that $\operatorname{Re} \xi_{0}>0$ induced by (A.21), then $\operatorname{Im} \xi_{0}$ and $\operatorname{Im} \tilde{\xi}_{0}^{2}=2 \operatorname{Re} \xi_{0} \operatorname{Im} \xi_{0}$ have the same sign. obtain

By taking the square root of (A.25) and (A.26), and inserting them into (A.24), we

$$
\left|\frac{1}{Z+\mathbf{i} \mu}\right| \leq C Z+\left|\frac{\mu}{\operatorname{Im} \xi^{2}}\right| \leq C
$$

where $C>0$ is a constant only depending on $k$ and $Z$. The proof is completed.

\section{Acknowledgements}

The research of X. Jiang is supported by China NSF grants Nos. 11771057, 11401040 and 11671052. The research of X. J. Li is supported by China NSF grant Nos. 11805049 and 11771440 and by the National Magnetic Confinement Fusion Science Program No. 2015GB110003.

\section{References}

[1] H. Ammari, G. BAO AND A. WOOD, An integral equation method for the electromagnetic scattering from cavities, Math. Method. Appl. Sci., 23 (2000), pp. 1057-1072.

[2] H. Ammari, G. BAO AND A. WOOD, Analysis of the electromagnetic scattering from a cavity, Japan J. Indust. Appl. Math., 19 (2002), pp. 301-310.

[3] H. Ammari, G. BAO AND A. WoOD, A cavity problem for Maxwell's equations, Meth. Appl. Anal., 9 (2002), pp. 249-260.

[4] G. BAO AND H. WU, Convergence analysis of the perfectly matched layer problems for timeharmonic Maxwell's equations, SIAM J. Numer. Anal., 43 (2005), pp. 2121-2143. 
[5] J. P. BÉRÉNGER, A perfectly matched layer for the absorption of electromagnetic waves, J. Comput. Phys., 114 (1994), pp. 185-200.

[6] J. H. BRAmble AND J. E. PASciaK, Analysis of a finite PML approximation for the three dimensional time-harmonic Maxwell and acoustic scattering problems, Math. Compt., 76 (2007), pp. 597-614.

[7] J. H. BRAMBLE AND J. E. PASCIAK, Analysis of a finite element PML approximation for the three dimensional time-harmonic Maxwell problem, Math. Comput., 77 (2008), pp. 1-10.

[8] J. H. Bramble, J. E. Pasciak And D. TRenev, Analysis of a finite PML approximation to the three dimensional elastic wave scattering problem, Math. Comput., 79 (2010), pp. 2079-2101.

[9] J. H. BRAmble AND J. E. PASCIAK, Analysis of a Cartesian PML approximation to the three dimensional electromagnetic wave scattering problem, Int. J. Numer. Anal. Model., 9 (2012), pp. 543-561.

[10] J. H. BRAmble AND J. E. PASCIAK, Analysis of a Cartesian PML approximation to acoustic scattering problems in $\mathbb{R}^{2}$ and $\mathbb{R}^{3}$, J. Comput. Appl. Math., 247 (2013), pp. 209-230.

[11] S. N. CHANDLER-WildE AND P. MONK, The PML for rough surface scattering, Appl. Numer. Math., 59 (2009), pp. 2131-2154.

[12] J. CHEN AND Z. CHEN, An adaptive perfectly matched layer technique for 3D time-harmonic electromagnetic scattering problems, Math. Comput., 77 (2008), pp. 673-698.

[13] Z. CHEN AND X. LIU, An adaptive perfectly matched layer technique for time-harmonic scattering problems, SIAM J. Numer. Anal., 43(2005), pp. 645-671.

[14] Z. CHEN AND H. WU, An adaptive finite element method with perfectly matched absorbing layers for the wave scattering by periodic structures, SIAM J. Numer. Anal., 41(2003), pp. 799-826.

[15] Z. CHEN AND X. WU, An adaptive uniaxial perfectly matched layer method for time-harmonic scattering problems, Numer. Math. Theor. Methods Appl., 1 (2008), pp. 113-137.

[16] Z. CHEN AND W. ZHENG, Convergence of the uniaxial perfectly matched layer method for timeharmonic scattering problems in two-layer media, SIAM J. Numer. Anal., 48 (2010), pp. 21582185.

[17] Z. CHEN, X. XIANG AND X. ZHANG, Convergence of the PML method for elastic wave scattering problems, Math. Comput., 85 (2016), pp. 2687-2714.

[18] Z. CHEN AND W. ZHENG, PML method for electromagnetic scattering problem in a two-layer medium, SIAM J. Numer. Anal., 55 (2017), pp. 2050-2084.

[19] W.-C. CHEW, Waves and Fields in Inhomogenous Media, Van Nodtrand Reimhold, New York, 1990.

[20] J. COYLE AND P. MONK, The Finite Element Approximation of Scattering in a Layered Medium, in Analytical and Computational Methods in Scattering and Applied Mathematics, F. Santosa and I. Stakgold, Eds., Chapman \& Hall/CRC, London. Research Notes in Mathematics number 417 (2000). pp. 67-84.

[21] M. DuRÁN, R. HEIN AND J. C. NÉdÉLEC, Computing numerically the Green's function of the half-plane Helmholtz operator with imedance boundary conditions, Numer. Math., 107 (2007), pp. 295-314.

[22] M. DuRÁn, I. MugA AND J. C. NÉDÉLEC, The Helmholtz equation in a locally perturbed halfplane with passive boundary, IMA J. Appl. Math., 71 (2006), pp. 853-876.

[23] M. DurÁn, I. MugA AND J. C. NÉDÉLEC, The Helmholtz equation in a locally perturbed halfspace with passive boundary, Arch. Rational Mech. Anal., 191 (2009), pp. 143-172.

[24] B. ENGQUIST AND A. MAJDA, Absorbing boundary conditions for the numerical simulation of waves, Math. Comput., 31 (1977), pp. 629-651.

[25] B. ENGQUIST AND A. MAJDA, Radiation boundary conditions for acoustic and elastic wave calcu- 
lations, Commun. Pure Appl. Math., 32 (1979), pp. 313-357.

[26] T. HAGSTROM, Radiation boundary conditions for the numerical simulation of waves, Acta Numer., 8 (1999), pp. 47-106.

[27] T. Hohage, F. SCHMidT AND L. ZsCHIEDRICH, Solving time-harmonic scattering problems based on the pole condition. II: Convergence of the PML method, SIAM J. Math. Anal., 35 (2003), pp. 547-560.

[28] X. JiAng, P. LI, J. LV AND W. ZHENG, An adaptive finite element PML method for the elastic wave scattering problem in periodic structure, ESAIM: Math. Model. Numer. Anal., 51 (2016), pp. 2017-2047.

[29] X. JIANG, P. LI, J. LV AND W. ZHENG, Convergence of the PML solution for elastic wave scattering by biperiodic structures, preprint.

[30] M. LASSAS AND E. SOMERSALO, On the existence and convergence of the solution of PML equations, Computing, 60 (1998), pp. 229-241.

[31] M. LASSAS AND E. SOMERSALO, Analysis of the PML equations in general convex geometry, Proceeding of the Royal Society of Edinburg (2001), pp. 1183-1207.

[32] F. L. TEIXEIRA AND W. C. CHEW, Advances in the theory of perfectly matched layers, in: W.C. Chew et al., (eds.), Fast and Efficient Algorithms in Computational Electromagnetics, pp. 283-346, Artech House, Boston, 2001.

[33] E. TURKel AND A. YEFET, Absorbing PML boundary layers for wave-like equations, Appl. Numer. Math., 27 (1998), pp. 533-557.

[34] D. YU, The Natural Boundary Integral Method and Its Applications, Science Press \& Kluwer Academic Publishers, 2002.

[35] D. ZHANG, F. MA AND H. DONG, A finite element method with rectangular perfectly matched layers for the scattering from cavities, J. Comput. Math., 27 (2009), pp. 812-834. 